\title{
Interacting hard-core bosons and surface preroughening
}

\author{
Alessandro Laio ${ }^{1,3}$, Giuseppe Santoro ${ }^{1,3}$, and Erio Tosatti ${ }^{1,2,3}$ \\ (1) International School for Advanced Studies (SISSA), Via Beirut 2, Trieste, Italy \\ (2) International Center for Theoretical Physics (ICTP), Strada Costiera, Trieste, Italy \\ (3) Istituto Nazionale per la Fisica della Materia (INFM), Via Beirut 2, Trieste, Italy
}

The theory of the preroughening transition of an unreconstructed surface, and the ensuing disordered flat (DOF) phase, is formulated in terms of interacting steps. Finite terraces play a crucial role in the formulation. We start by mapping the statistical mechanics of interacting (up and down) steps onto the quantum mechanics of two species of one-dimensional hard-core bosons. The effect of finite terraces translates into a number-non-conserving term in the boson Hamiltonian, which does not allow a description in terms of fermions, but leads to a two-chain spin problem. The Heisenberg spin-1 chain is recovered as a special limiting case. The global phase diagram is rich. We find the DOF phase is stabilized by short-range repulsions of like steps. On-site repulsion of up-down steps is essential in producing a DOF phase, whereas an off-site attraction between them is favorable but not required. Step-step correlation functions and terrace width distributions can be directly calculated with this method. 


\section{INTRODUCTION}

The surface roughening transition and the nature of the rough phase are theoretically very well understood by a variety of approaches ranging from phenomenological descriptions, based on the sine-Gordon model, to microscopic solid-on-solid (SOS) models. [1]

The preroughening transition (PR) and the ensuing disordered flat phase (DOF), both predicted several years ago by Rommelse and den Nijs, [2] have also been studied and characterized within certain restricted solid-on-solid (RSOS) models. [3 9] Although the physics behind these models, and the ingredients stabilizing the DOF phase, have been discussed in some detail, it is still useful to explore this subject from a different, and perhaps more physically appealing, perspective. The RSOS models, in particular, do not directly emphasize steps, terraces, and kinks, which on the other hand are very crucial actors in these transitions. This need is made more urgent by the recent experimental evidences for preroughening on rare gas solid (111) surfaces, 10, 11] which calls for a detailed reinvestigation of the step-step interactions, [8] or the reconstructive tendencies, [5.6, 6],9] crucial to obtain a DOF phase.

In the context of roughening, Villain and Vilfan, 12 14] den Nijs, 15] and Balents and Kardar, 16] for the case of reconstructed surfaces, and Villain, Grempel, and Lapujoulade, [17] for the case of vicinal surfaces, have shown that a more phenomenological approach based on working directly with steps yields a very direct picture of the physics involved.

Anisotropic surfaces, in particular, have a definite direction of stronger bonding along which a step tends to run; kinks on such steps, involving the breaking of strong bonds, are energetically expensive. The (110) face of fcc noble metals $(\mathrm{Au}, \mathrm{Pt}, \mathrm{Ag}, \ldots)$ is a physical realization of an anisotropic surface, with a stronger, compact, [11̄0] direction, and a softer [001] direction. In the strongly anisotropic limit, the transfer matrix problem for the system of steps can be mapped onto the imaginary-time evolution of a system of quantum particles in one dimension (1D), the imaginarytime being the preferred direction in which the steps run. 18 20] This is a well known mapping, heavily exploited, for instance, in the theory of uniaxial commensurate-to-incommensurate transitions of adsorbates. [20,19]

Following a line of thought initiated by Ref. [13], Balents and Kardar applied the full machinery of the theory of interacting fermions in one dimension to explore the possible phase diagrams of generic $(p \times 1)$ reconstructed anisotropic surfaces. [16] In their approach, steps of double height are forbidden (as energetically too expensive), while up and down monoatomic steps are mapped onto spin- $1 / 2$ fermions in $1 \mathrm{D}$, described (in the continuum limit) by the hamiltonian 16$]$

$$
H=\sum_{\sigma} \int d x \psi_{\sigma}^{\dagger}\left(-\frac{\gamma}{2} \partial_{x}^{2}-\mu\right) \psi_{\sigma}+\sum_{\sigma \sigma^{\prime}} \int d x d x^{\prime} n_{\sigma}(x) V_{\sigma \sigma^{\prime}}\left(x-x^{\prime}\right) n_{\sigma^{\prime}}\left(x^{\prime}\right) .
$$

Here $\gamma$ is the inverse line tension of a step, $V_{\sigma \sigma^{\prime}}(x)$ is an interaction between steps, and the remaining notation is standard. This Hamiltonian describes infinite steps, traversing the entire length of the sample, as the number of particles is conserved by the (imaginary-time) dynamics. In reality, steps on surfaces can lead to finite defects by forming loops (i.e., finite terraces) on the surface. [13,16] The order of the reconstruction $p$, dictates, through the symmetry of the different ground states, the form of the "loop" terms which are allowed. [13, 16] For a $(p \times 1)$ reconstructed surface, the Hamiltonian $H$ has to be supplemented with a term of the type

$$
H_{\mathrm{LOOP}}=\lambda \int d x\left[\prod_{k=0}^{p-1} \psi_{\uparrow}(x+k a) \psi_{\downarrow}(x+k a)+\text { H.c. }\right],
$$

where $a$ is a cut-off distance of the order of the lattice constant. Balents and Kardar argued, by power counting, that the effect of finite terraces, i.e., the introduction of $H_{\mathrm{LOOP}}$, is irrelevant for $p>2$, marginal for $p=2$, and strongly relevant for $p=1$. They went on by addressing in detail the $p=2$ case, of relevance to the (110) missing-row reconstructed facet of Au. (See also Ref. [15] for closely related work on the $p=2$ case.) However, the unreconstructed $(p=1)$ case was not pursued further.

The approach we take in the present paper is similar in spirit. Our specific goal, however, is to address the question of the presence of a DOF phase, and the preroughening transition, for unreconstructed surfaces. Thus, in the classification introduced above, we are now interested in detail in the $p=1$ case. Technically, this leads, as we shall see, to significant differences with respect, for instance, to Ref. [16], and to a new phase diagram quite different from the $p>1$ cases.

The crucial point is that for $p=1$ the loop terms (see Eq. (2)) are of the BCS-like form $\lambda \int d x\left(\psi_{\uparrow}(x) \psi_{\downarrow}(x)+\right.$ H.c.), i.e., a strongly relevant one-body piece. This might appear as just a minor complication, at first glance, since quadratic terms can be easily diagonalized by a Bogoliubov transformation. Closer consideration, however, leads to reconsider the whole mapping. Fermionic minus signs have no role whatsoever in the mapping of a classical statistical mechanics 
problem. The natural statistics to use is always the bosonic. In the present case, a hard-core constraint will be necessary, in order to implement the appropriate configurational space (for instance, the non-crossing constraint for steps of the same type). In a one-dimensional quantum problem, the choice of the statistics is, quite often, not a big problem, as we can transform, by a Wigner-Jordan transformation, hard-core bosons into fermions, with a transformed hamiltonian which has exactly the same form (only boundary conditions have to be considered carefully). In our case, however, pairing terms of the type $a_{i, \uparrow} a_{i+1, \downarrow}$, which (see below) are essential to describe finite terraces, do not transform into simple fermionic BCS-like terms, and become non-local after a Wigner-Jordan transformation. This will force us to work with hard-core bosons.

Our approach, in summary, is as follows. We assume, as in Ref. [16], that the only relevant extended defects are monoatomic steps, which can be either up or down. Steps of the same kind are forbidden to cross, while steps of different type can cross. Moreover, steps interact with each other, have kinks, and can form finite terraces on the surface. These steps are then mapped onto world-lines of hard-core bosons in one dimension. Kinks on the steps correspond to hopping terms in the quantum Hamiltonian. Pairs of up-down steps which are created and annihilated to form finite terraces on the surface, give number-non-conserving terms in the quantum Hamiltonian. 20] Pairwise interactions between the steps are taken into account by corresponding two-body terms in the quantum model.

The present work is concerned with the case of a low-index unreconstructed surface. Extensions to the case of vicinals, for which the long-ranged nature of the step-step interactions is an essential ingredient, are left to a future study.

Our main goals in working out this type of approach to PR are the following: (a) to build a formulation providing a more direct access to the physics of PR, which is somewhat hidden in the RSOS formulations; (b) to explore more directly the role of step-step interactions; (c) to study step-step correlation functions and terrace width distributions, not available so far. As it turns out, we have found that this approach is quite successful on all three accounts.

The paper is organized as follows. In Sec. II we present in detail the classical statistical mechanics model of interacting steps, which is then mapped onto the corresponding 1D quantum model of hard-core bosons in Sec. III. In Sec. IV we consider in detail the spin-1 limit, obtained by setting the on-site step-step repulsion to infinity, and then map the general case to a problem of two spin-1/2 Heisenberg chains. Sec. $\mathrm{V}$ contains a summary of bosonization plus finite-size scaling calculations done in order to extract the phase diagram of the model. Sec. VI summarizes the relevant order parameters and correlation functions investigated. In Sec. VII we present our results for the overall phase diagram of the model. Sec. VIII illustrates our results for the step-step correlations and the terrace width distributions. Finally, Sec. IX contains a discussion of the results and some conclusive remarks.

\section{THE MODEL}

We assume the only relevant extended defects involved in the surface PR transition to be steps, which can be either up or down. These steps interact with each other, they can have kinks, and they can form finite terraces on the surface. Fig. 1 shows a schematic picture of a surface with steps.

Our model will be defined on a square lattice, and we will assume the steps to run preferentially in one direction (the vertical direction in Fig. 1). The steps are only allowed to make simple nearest neighbor kinks. Steps running in the horizontal direction are assumed to be energetically expensive and neglected. Hence, our surface is, by construction, very highly anisotropic.

We define the model by assigning its transfer matrix along y. Denoting by $|S(j)\rangle$ and $|S(j+1)\rangle$ the configuration of the $j$-th and $j+1$-th horizontal strips, we have

$$
\begin{gathered}
\langle\mathrm{S}(\mathrm{j}+1)|\mathcal{T}| \mathrm{S}(\mathrm{j})\rangle= \\
=\exp \left(-\beta\left(\delta_{S} N_{S}^{(j)}+\delta_{K} N_{K}^{(j, j+1)}+\sum_{s=0,1} \delta_{T_{s}} N_{T_{s}}^{(j, j+1)}+\delta_{e x} N_{e x}^{(j, j+1)}+V_{\text {step-step }}\right)\right)
\end{gathered}
$$

where $\beta$ is the inverse temperature, and (see also Fig. 2):

- $\delta_{S}$ represents the energy cost (per unit length) of a step running along the y-direction; $N_{S}^{(j)}$ is the number of steps in the strip $j$;

- $\delta_{K} N_{K}^{(j, j+1)}$ is the energy cost of $N_{K}^{(j, j+1)}$ kinks between strip $j$ and $j+1$;

- $\delta_{T_{s}} N_{T_{s}}^{(j, j+1)}$ is the energy cost for the creation of $N_{T_{s}}^{(j, j+1)}$ terraces of "size" $s$ between strip $j$ and $j+1$. We will always assume $s=1$, or 0 (see Fig. 2). 
- $\delta_{e x} N_{e x}^{(j, j+1)}$ is the energy associated to the crossing of $N_{e x}^{(j, j+1)}$ pairs of opposite steps between strip $j$ and $j+1$;

- $V_{\text {step-step }}=V^{\|}+V^{\perp}$, with $V^{\|}$and $V^{\perp}$ describing respectively the interaction between steps of the same kind and of the opposite kind. For $V^{\|}$, we assume a generic repulsive interaction

$$
V^{\|}=\sum_{\sigma} \sum_{k>i} \widetilde{V}_{k-i}^{\|} n_{i, \sigma} n_{k, \sigma}
$$

with $V_{k-i}^{\|}$possibly possessing an elastic long-range tail of the form $\approx|k-i|^{-2}$. Here $n_{i, \uparrow(\downarrow)}$ is 1 if there is a step up (down) at site $i$. Similarly, we assume $V^{\perp}$ to be given by

$$
V^{\perp}=\widetilde{V}_{0}^{\perp} \sum_{i} n_{i, \uparrow} n_{i, \downarrow}+\sum_{\sigma} \sum_{k>i} \widetilde{V}_{k-i}^{\perp} n_{i, \sigma} n_{k, \bar{\sigma}}
$$

The sign of the terms in $V^{\perp}$, particularly at short-range, depends on microscopic details and need not be specified at this stage.

If we assume periodic boundary conditions in $y$ direction, i.e., $\left|\mathrm{S}\left(\mathrm{N}_{\mathrm{y}}+1\right)\right\rangle=|\mathrm{S}(1)\rangle$, the partition function of this system is

$$
Z=\lim _{N_{y} \rightarrow \infty} \operatorname{Tr} \mathcal{T}^{\mathrm{N}_{\mathrm{y}}}
$$

\section{THE QUANTUM MODEL}

It is well known that, in the strong anisotropy (or time-continuum) limit, many $D$ dimensional classical problems can be mapped onto $D-1$ dimensional quantum problems. [18] This relationship is established by means of the path integral formalism. In particular, the up and down steps are mathematically equivalent to the world lines of spin-up and spin-down hard-core bosons, and the preferential direction in which the steps run plays the role of time in the quantum problem. The hard-core condition is imposed in order to implement the non-crossing condition for steps of the same type, a physically justified restriction, in view of the large energetic cost of double-step regions. The non-crossing constraint for steps of the same type would be automatically satisfied by the Pauli principle if we were to deal with spin- $1 / 2$ fermions. (See below for more comments on the problem of quantum statistics.)

We consider the following quantum Hamiltonian

$$
\begin{gathered}
H=-t^{\|} \sum_{i, \sigma}\left(a_{i, \sigma}^{\dagger} a_{i+1, \sigma}+\text { H.c. }\right)-\mu \hat{N}+ \\
-t_{0}^{\perp} \sum_{i}\left(a_{i, \uparrow}^{\dagger} a_{i, \downarrow}^{\dagger}+\text { H.c. }\right)-t_{1}^{\perp} \sum_{i, \sigma}\left(a_{i, \sigma}^{\dagger} a_{i+1, \bar{\sigma}}^{\dagger}+\text { H.c. }\right)-t_{e x} \sum_{i, \sigma} a_{i+1, \bar{\sigma}}^{\dagger} a_{i, \sigma}^{\dagger} a_{i+1, \sigma} a_{i, \bar{\sigma}}+ \\
+\sum_{\sigma} \sum_{j>i} V_{j-i}^{\|} n_{i, \sigma} n_{j, \sigma}+V_{0}^{\perp} \sum_{i} n_{i \uparrow} n_{i \downarrow}+\sum_{\sigma} \sum_{j>i} V_{j-i}^{\perp} n_{i, \sigma} n_{j, \bar{\sigma}}
\end{gathered}
$$

with $a_{i, \sigma}$ representing the destruction operator for a spin $\sigma$ hard-core boson and with $\hat{N}=\hat{N}_{\uparrow}+\hat{N}_{\downarrow}$ the total number of particles. We will work in the subspace $\hat{N}_{\uparrow}=\hat{N}_{\downarrow}$, for a low-index surface. (For a vicinal surface of angle $\phi$, we would have $\left(\hat{N}_{\downarrow}-\hat{N}_{\uparrow}\right)=L \tan \phi$.)

Within a path-integral approach, it can be shown 18 that the ground state properties of this quantum Hamiltonian correspond to the temperature properties of the classical step model, whose transfer matrix is given by Eq. (3), in the large anisotropy limit. Specifically, the classical parameters turn out to be given by:

$$
\begin{aligned}
\epsilon t^{\|} & =e^{-\beta \delta_{K}}, \quad \epsilon t_{0,1}^{\perp}=e^{-\beta \delta_{T_{0,1}},} \quad \epsilon t_{e x}=e^{-\beta \delta_{e x}} \\
\epsilon(-\mu) & =\beta \delta_{S}, \quad \epsilon V_{i-j}^{(\|, \perp)}=\beta \tilde{V}_{i-j}^{(\|, \perp)}
\end{aligned}
$$


where $\epsilon$ is the Trotter discretization time for the quantum path-integral.

The mapping is asymptotically correct only in the limit $\epsilon \rightarrow 0$. This a) implies, clearly, a strong anisotropy limit for the classical problem and b) does not allow a straightforward identification of a classical low- or high-temperature limit. Indeed, if all the parameters of the quantum problem are of order one, taking $\beta \rightarrow 0$ or $\beta \rightarrow \infty$ makes Eq. (8) incompatible with the requirement that the left-hand side should be a small quantity (of order $\epsilon$ ). In other words, the mapping is justified so long as

$$
\text { Kinetic couplings }=\left(\delta_{K}, \delta_{T_{0}}, \cdots\right) \gg T \gg\left(\delta_{S}, \tilde{V}, \cdots\right)=\text { Potential couplings },
$$

and nothing can be said, in principle, about the infinite temperature limit. This should be always kept in mind when considering the infinite temperature limit from the quantum model point of view (see, e.g., sec. VIIB).

It is also worth stressing that the (hard-core) boson statistics of the $a$ operators in the Hamiltonian is crucial to the nature of the phases and transition lines in the phase diagram. Indeed, unlike other terms in the Hamiltonian, the terrace creation terms cannot be translated into simple (i.e., local) fermionic BCS-like terms by a Jordan-Wigner transformation. In such instances, the correct statistics to use is undoubtedly the bosonic one, as fermionic minus signs do not appear in a classical statistical mechanics problem. This point seems to be not always appreciated in the literature. [28]

We now make contact with previous work in the context of surface physics. The model in Eq. (7), with $t_{e x}=0$ and $t_{0,1}^{\perp}=0$, has been considered, in its continuum version, by Balents and Kardar. [16] (See also Refs. [12] and [15] for related work.) In the absence of $t^{\perp}$-terms, particles are taken to be fermions. The emphasis of Ref. [16] was on $(p \times 1)$ reconstructed surfaces, particularly with $p=2$. The effect of finite terraces, i.e., closed loops of steps, was argued to be irrelevant for $p>2$, marginal for $p=2$, and strongly relevant for $p=1$. [16] The unreconstructed $(p=1)$ case, however, was not pursued at all. As just argued, the $p=1$ case cannot be tackled in terms of fermions. The effect of the finite terraces on an unreconstructed surface - the $t^{\perp}$ terms in Eq. (7) - is one of the points addressed in detail in the present work. Moreover, we show that restricting the analysis to a simple Hubbard-type on-site interaction does not lead to the full richness of the phase diagram; nearest-neighbor interactions are essential in order to stabilize, for instance, a DOF phase.

\section{CANONICAL TRANSFORMATIONS}

\section{A. $V_{0}^{\perp}=\infty$ : Mapping to a spin-1 chain.}

For a special choice of the parameters, the model in Eq. (7) reduces to a well studied problem. Consider the case in which both $V^{\|}$and $V^{\perp}$ are truncated to nearest neighbors, $V_{j-i}^{\|, \perp}=V_{1}^{\|, \perp} \delta_{j, i+1}$, in the limit of infinite on-site repulsion of opposite steps, $V_{0}^{\perp} \rightarrow \infty$. The limit $V_{0}^{\perp} \rightarrow \infty$ enforces, in absence of $t_{e x}$, a non-crossing condition for opposite steps as well, and allows only three states per site, which we can easily map onto a spin-1 variable as follows:

$$
\begin{aligned}
\text { step up } & a_{i, \uparrow}^{\dagger}|0\rangle & =\mid S_{i}^{z} & =+1\rangle \\
\text { no step } & |0\rangle & =\mid S_{i}^{z} & =0\rangle \\
\text { step down } & a_{i, \downarrow}^{\dagger}|0\rangle & =\mid S_{i}^{z} & =-1\rangle .
\end{aligned}
$$

It is then straightforward to show that all possible matrix elements of $H$ in Eq. (7) coincide exactly with those of the spin-1 Heisenberg chain

$$
H_{\text {Heis }}=-\frac{J_{x y}}{2} \sum_{i}\left(S_{i}^{+} S_{i+1}^{-}+\text {H.c. }\right)+J_{z} \sum_{i} S_{i}^{z} S_{i+1}^{z}+D \sum_{i}\left(S_{i}^{z}\right)^{2}
$$

if the following parameter choice is made:

$$
t^{\|}=t_{1}^{\perp}=J_{x y}, \quad t_{e x}=0, \quad V_{1}^{\|}=-V_{1}^{\perp}=J_{z}, \quad-\mu=D .
$$

A nonzero $t_{e x}$ term, when present, translates into a quartic spin term

$$
-t_{e x} \sum_{i}\left[\left(S_{i}^{+}\right)^{2}\left(S_{i+1}^{-}\right)^{2}+\text { H.c. }\right]
$$


and will be shown to be relevant in stabilizing the rough phase for finite repulsive $V_{1}^{\|}$. The phase diagram for this special case, in the surface physics relevant region $-\mu=D>0$, can be directly borrowed from the literature. [3, 21]

The Heisenberg spin- 1 chain was also obtained by another route, namely by the quantum mapping of RSOS models, by den Nijs and Rommelse, who gave a very detailed discussion of the surface physics interpretation of the different phases. [3] The region in which a DOF phase is stabilized is found for $J_{z}>0$, i.e., it corresponds to repulsive $V_{1}^{\|}$and attractive $V_{1}^{\perp}$ (see Eq. (11)). The latter condition is, however, not crucial, as we shall see later on. The flatness of the DOF phase is directly related to the Haldane gap in the spin-1 chain. [3] If the interactions are much bigger than the cost of a unit of step $\left(J_{z} \gg D\right.$, see line (a) in Fig. 3), at low temperatures (i.e., for large $\left.J_{z} / J_{x y}\right)$ the system will reconstruct into an ordered sequence of up-down steps (the Néel phase of the spin- 1 chain). By increasing temperature (i.e., lowering $\left.J_{z} / J_{x y}\right)$ the system undergoes an Ising transition to a DOF phase, in which the positional order of the up-down sequence of steps is lost. [3] If the cost of the unit step is larger than the interactions, $\left(J_{z} \ll D\right.$, see line (b) in Fig. 3), the low temperature phase is flat, and the (preroughening) transition to the DOF phase has non-universal exponents. [3]

If we impose the condition $V_{0}^{\perp} \rightarrow \infty$ without assuming Eq. (11), what we are considering is always a three-stateper-site problem, but the resulting Hamiltonian does not have the simple bilinear form (10) in terms of the spin-1 operators. We will refer to this general case as a spin-1 chain. The specific case in Eq. (10) will be referred to as Heisenberg spin-1 chain.

\section{B. The general case: Mapping to two spin-1/2 chains}

In order to study the model in Eq. (7) for more general parameter values, in particular for $V_{0}^{\perp} \neq \infty$, it is convenient to abandon the spin- 1 representation and map onto a problem of two coupled spin- $1 / 2$ chains, where the total number of states per rung is four, instead of three. Introducing the usual spin- $1 / 2$ representation for each of the two species of hard-core bosons,

$$
a_{i, \uparrow(\downarrow)}^{\dagger}=S_{i, 1(2)}^{+}, \quad n_{i, \uparrow(\downarrow)}=S_{i, 1(2)}^{z}+\frac{1}{2},
$$

and performing a $\pi$-rotation for the $\mathbf{S}_{i, 2}$ spins around the x-axis, which amounts to a particle-hole transformation for the down-bosons, $S_{i, 2}^{ \pm} \rightarrow S_{i, 2}^{\mp}$ and $S_{i, 2}^{z} \rightarrow-S_{i, 2}^{z}$, one can rewrite the Hamiltonian (7) as the following model of two spin- $1 / 2$ chains ( $\alpha=1,2$ denoting the chain) with opposite magnetic fields:

$$
\begin{aligned}
H= & -t^{\|} \sum_{i, \alpha}\left(S_{i, \alpha}^{+} S_{i+1, \alpha}^{-}+\text {H.c. }\right)+V_{1}^{\|} \sum_{i, \alpha} S_{i, \alpha}^{z} S_{i+1, \alpha}^{z}+h \sum_{i}\left(S_{i, 1}^{z}-S_{i, 2}^{z}\right) \\
& -t_{0}^{\perp} \sum_{i}\left(S_{i, 1}^{+} S_{i, 2}^{-}+\text {H.c. }\right)-V_{0}^{\perp} \sum_{i} S_{i, 1}^{z} S_{i, 2}^{z}+ \\
& -t_{1}^{\perp} \sum_{i, \alpha}\left(S_{i, \alpha}^{+} S_{i+1, \bar{\alpha}}^{-}+\text {H.c. }\right)-V_{1}^{\perp} \sum_{i, \alpha} S_{i, \alpha}^{z} S_{i+1, \bar{\alpha}}^{z}+ \\
& -t_{e x} \sum_{i}\left(S_{i+1,1}^{+} S_{i, 1}^{-} S_{i+1,2}^{+} S_{i, 2}^{-}+\text {H.c. }\right)
\end{aligned}
$$

where, for simplicity, we have considered only interaction terms up to nearest neighbors and we have omitted the constant $\frac{1}{4}\left[V^{\perp}(q=0)+V^{\|}(q=0)\right]$ (with $V^{\|(\perp)}(q)$ we denote the Fourier transforms of the potentials, and $V^{\|(\perp)}(q=$ $\left.0)=\sum_{j} V_{j-i}^{\|(\perp)}\right)$. The magnetic field $h$ is related to the chemical potential $\mu$ in the following way:

$$
h=-\mu+\frac{1}{2}\left[V^{\perp}(q=0)+V^{\|}(q=0)\right]=-\mu+\left(V_{0}^{\perp} / 2+V_{1}^{\perp}+V_{1}^{\|}\right) .
$$

Notice that after the spin-rotation, performed to get a standard $S_{1}^{+} S_{2}^{-}$coupling between the chains starting from the $a_{\uparrow}^{\dagger} a_{\downarrow}^{\dagger}$ boson term, the signs of the $V^{\perp}$ terms are all changed, since $S_{i, 2}^{z} \rightarrow-S_{i, 2}^{z}$. For the same reason, the chemical

potential terms transform into opposite magnetic fields for the two chains. The condition $\hat{N}_{\uparrow}=\hat{N}_{\downarrow}$ reads, after the canonical transformation, as zero total magnetization for the spins, $\sum_{i}\left(S_{i, 1}^{z}+S_{i, 2}^{z}\right)=0$. 222] 


\section{LOW-ENERGY HAMILTONIAN FROM FINITE-SIZE DIAGONALIZATION}

With a well defined quantum spin chain problem at hand, it is standard practice to study its phase diagram by a combination of field-theoretical arguments and finite-size exact diagonalization data.

At weak coupling, a standard field-theory approach to one dimensional quantum systems consists in applying bosonization techniques. This was done by Strong and Millis for the case of two coupled spin-1/2 Heisenberg chains, i.e., a special case of Eq. (13) with $t_{1}^{\perp}=V_{1}^{\perp}=0, t_{e x}=0, h=0$, and $V_{0}^{\perp}=2 t_{0}^{\perp}=-J_{K}$. [23] The procedure can be easily extended to our case. Introducing symmetric and antisymmetric combinations of the bosonic phase fields, $\Theta_{N, S}$ and $\Theta_{J, S}$, 23,24] which represent the bosonic sound-like excitations of the system in the gapless phase, the low-energy Hamiltonian reads:

$$
\begin{gathered}
H=\sum_{\alpha=S, A} \frac{v_{\alpha}}{4 \pi} \int d x\left[\frac{1}{K_{\alpha}}\left(\nabla \Theta_{J, \alpha}\right)^{2}+K_{\alpha}\left(\nabla \Theta_{N, \alpha}\right)^{2}\right]+ \\
-\frac{1}{(\pi a)^{2}} \int d x\left\{\cos \left(\Theta_{N, A}\right)\left\{A+B\left[\cos \left(2 \Theta_{J, S}\right)+\cos \left(2 \Theta_{J, A}\right)\right]\right\}+C \cos \left(2 \Theta_{J, S}\right)+D \cos \left(2 \Theta_{J, A}\right)\right\},
\end{gathered}
$$

where $a$ is a short distance cut-off, and $A, B, C$, and $D$ are coupling dependent (but cut-off independent) constants.

Let us consider the A-sector first. The A-sector can be gapless only if $1 / \sqrt{K_{A}}>2$ and, simultaneously, $2 \sqrt{K_{A}}>2$.

This is, of course, impossible. Thus, the A-sector flows to strong coupling and develops a gap. [23]

The Hamiltonian for the S-sector, renormalized by the A-sector, will be of the form:

$$
H_{S}=\frac{v_{S}}{4 \pi} \int d x\left[\frac{1}{K_{S}}\left(\nabla \Theta_{J, S}\right)^{2}+K_{S}\left(\nabla \Theta_{N, S}\right)^{2}\right]-\frac{V_{S}}{(\pi a)^{2}} \int d x \cos \left(2 \Theta_{J, S}\right) .
$$

The cosine term is relevant and opens up a gap when $K_{S}<1$. Thus the system undergoes a KT transition when $K_{S} \rightarrow 1$. As discussed in the following sections, this is associated to a roughening transition.

If the symmetric sector flows to the free Klein-Gordon Hamiltonian (i.e., the Luttinger model) in some range of parameters, the low energy spectrum of the two chains, for $L \rightarrow \infty$, will have the form of a spinless Luttinger model describing symmetric excitations. Expressing $\Theta_{J(N), S}$ in terms of bosonic creation operators we can write: 24].

$$
H_{S}=v_{S} \sum_{k}|q| b_{k}^{\dagger} b_{k}+\frac{\pi}{4 L}\left(v_{N} N_{S}^{2}+v_{J} J_{S}^{2}\right)
$$

where $N_{S}$ and $J_{S}$ are the symmetric sector total number $(N)$ and current $(J)$ operators, $v_{S}$ is the renormalized sound velocity, $v_{N}=v_{S} / K_{S}$, and $v_{J}=K_{S} v_{S}$. 24]

In order to compute $v_{N}$, we note that the simplest charge excitation not involving the current part consists in adding 2 particles to the system. Thus we have:

$$
v_{N}(L)=\frac{L}{\pi}[E(\Delta N=2)-E(0)] .
$$

To compute $v_{J}$, notice that if a magnetic flux $\Phi$ is concatenated with the ring, the current part of the energy spectrum is modified in this way:

$$
\frac{\pi}{4 L} v_{J} J_{S}^{2} \longrightarrow \frac{\pi}{4 L} v_{J}\left(J_{S}+4 \frac{\Phi}{\Phi_{0}}\right)^{2}
$$

( $\Phi_{0}$ is the elementary flux quantum). Therefore:

$$
v_{J}(L)=\frac{L}{8 \pi} \frac{\partial^{2} E(\Phi)}{\partial\left(\Phi / \Phi_{0}\right)^{2}} .
$$

Finally, $v_{S}$ can be computed from

$$
v_{S}(L)=\frac{L}{2 \pi}[E(k=2 \pi / L)-E(0)]
$$

where $E(0)$ is the ground state energy and $E(k=2 \pi / L)$ is the energy of the lowest excited state of momentum $k=2 \pi / L$.

As a consequence, $K_{S}$ can be equivalently computed from the finite-size extrapolation of $v_{J}(L) / v_{S}(L)$, of $v_{S}(L) / v_{N}(L)$, or of $\sqrt{v_{J}(L) / v_{N}(L)}$. If the finite-size data are compatible with a Luttinger Liquid picture, i.e., with a spectrum of the form (14), then these three extrapolations should converge, as $L \rightarrow \infty$, to a single value. 


\section{ORDER PARAMETERS}

We now define the order parameters and correlation functions we have to consider in order to study the phase diagram of our model. We will basically deal with four correlation functions, whose behavior in the different phases is summarized in Tab. 1.

- The height-height correlation, defined by $G_{h}(r)=\left\langle\left(h_{r}-h_{0}\right)^{2}\right\rangle$, diverges logarithmically as

$$
G_{h}(r)=\frac{2 K}{\pi^{2}} \ln (r)+\ldots
$$

in the rough phase, with $K \geq 1$, while it remains limited in the flat, Néel, and DOF phases. [25] At the roughening transition, $K$ takes the universal value of 1 [1].15]. This gives a simple criterion for determining whether a phase is rough or not. In fact, the coefficient $K$ coincides with the Luttinger exponent $K_{S}$ for the symmetric sector, [16] which can be extracted by finite-size scaling of exact diagonalization data (cfr. Sec. V).

- The string correlation function, defined by [3]

$$
G_{s}(r)=-\left\langle\left(S_{0}^{z} \exp \left(i \pi \sum_{j=1}^{r-1} S_{j}^{z}\right) S_{r}^{z}\right\rangle .\right.
$$

(We introduced the notation $S_{i}^{z}=n_{i \uparrow}-n_{i \downarrow}$.) The phase factor contributes a plus (minus) sign if there are an even (odd) number of steps between site 0 and site $r$. In the DOF and in the Néel phases, a step up (down) is preferentially followed by a step down (up). In these configurations, $G_{s}(r)$ gets a contribution equal to 1 every time sites 0 and $r$ are occupied by a step. Thus, in the DOF phase and in the Néel phase $G_{s}(r)$ decays exponentially to the square of the mean density of steps. [15]

- The staggered magnetization, defined by

$$
\mathcal{N}=\lim _{L \rightarrow \infty} \frac{1}{L} \sum_{j}(-1)^{j}\left\langle S_{0}^{z} S_{j}^{z}\right\rangle .
$$

A Néel phase will be signaled by a non-zero staggered magnetization $\mathcal{N}$, while $\mathcal{N}$ is zero in the rough, DOF and flat phases.

- The flatness order parameter, defined by [3]

$$
\mathcal{F}=\lim _{L \rightarrow \infty} \frac{1}{L} \sum_{r}\left\langle\exp \left(i \pi \sum_{j=1}^{r} S_{j}^{z}\right)\right\rangle .
$$

$\mathcal{F}$ has a non zero value only in the ordered flat phase (in the DOF phase the exponential fluctuates between 1 and -1 as $r$ is increased).

\begin{tabular}{|l|l|l|l|l|}
\hline & Flat & Rough & DOF & Néel \\
\hline$K_{S}$ & gapped & $>1$ & gapped & gapped \\
\hline$G_{h}(r)$ (for large $\left.r\right)$ & $<\infty$ & $\rightarrow \frac{2 K_{S}}{\pi^{2}} \ln (r)$ & $<\infty$ & $<\infty$ \\
\hline$G_{S}(r)$ (for large $\left.r\right)$ & $\rightarrow 0$ & $\rightarrow 0$ & $\rightarrow\left\langle\left(S^{z}\right)^{2}\right\rangle$ & $\rightarrow\left\langle\left(S^{z}\right)^{2}\right\rangle$ \\
\hline $\mathcal{N}$ & $=0$ & $=0$ & $=0$ & $\neq 0$ \\
\hline $\mathcal{F}$ & $>0$ & 0 & 0 & 0 \\
\hline
\end{tabular}




\section{THE OVERALL PHASE DIAGRAM}

Our model, even if the interactions are truncated to first neighbors, contains many parameters. Rather than trying to describe the phase diagram in an exhaustive form, we will focus our discussion on a few questions, that we consider quite relevant with respect to the surface physics interpretation of our model. This will take us to consider in detail some special planes cut through the phase diagram, and will also give us an idea of its global structure.

Let us consider, once again, the Heisenberg spin-1 phase diagram in Fig. 3. From the surface physics point of view, it presents some unpleasant features: since the increasing temperature curve, for a given surface, is a line through the origin (the origin corresponds to the infinite $T$ point; cfr. Eq. (8)), every "surface" with repulsive interaction between steps of the same kind $\left(V_{1}^{\|}>0\right)$ has a preroughening transition at finite temperature, and no rough phase at finite $T$. On the other hand, if $V_{1}^{\|}$is attractive there is only roughening.

In relation to these problems, we will discuss the following main questions:

A Is the attractive $V_{1}^{\perp}$ term between opposite sign steps essential in order to stabilize a DOF phase?

B Is there a choice of the parameters for which our model can describe a surface with a finite roughening temperature?

C How does the presence or absence of a preroughening transition depend on the relative strength of the step-step interactions to the cost per unit length of a step.

D What is the role of the opposite step on-site repulsion $V_{0}^{\perp}$.

In the following we address these points directly.

\section{A. The role of attraction between opposite steps: Spin-1 chain with $V_{1}^{\perp}=0$.}

In order to explore the different roles of the two interactions $V_{1}^{\perp}$ and $V_{1}^{\|}$, we have first studied the effect of a repulsive $V_{1}^{\|}$, keeping $V_{1}^{\perp}=0$. For the time being, we still work with the spin-1 condition, i.e., we impose an infinite on-site repulsion of opposite steps $\left(V_{0}^{\perp}=\infty\right)$.

For this choice of parameters, we do not find any point with $V_{1}^{\|}>0$ in which the finite-size data might indicate a vanishing gap. This is compatible with the results of den Nijs and Rommelse about the location of the KT-transition in the Heisenberg spin-1 phase diagram. [3] The system undergoes a roughening transition only at infinite temperature.

In Fig. 4 we draw a qualitative phase diagram for values of the parameters $\mu$ and $V^{\|}$which are relevant for surface physics (i.e., positive energy cost for a step, and repulsive interaction between steps of the same kind). It is quite remarkable how the DOF phase survives the turning off of the attraction between steps of the same kind. As a matter of fact, taking $V_{1}^{\perp}=0$ leads to the disappearance of the reconstructed (Néel) phase from the physically interesting region of the phase diagram, and, therefore, to an even larger DOF phase. (We will further discuss the roles of $V_{1}^{\perp}$ and $V_{1}^{\|}$in stabilizing the DOF phase later in this Section).

\section{B. Infinite or finite roughening temperature? The role of $t_{e x}$.}

In order to discuss the point concerning the roughening temperature, we observe that, as $T \rightarrow \infty$, the kinetic terms tend to be the only relevant pieces of the Hamiltonian, see Eq. (8). Thus, we now consider the model in absence of potential terms $(V)$, and for zero chemical potential $\mu$. For the time being, we also take $t_{e x}=0$. The crucial role of the $t_{e x}$-term will be discussed afterwards. In this case, the Hamiltonian reduces to that of two coupled XY chains:

$$
H=-t^{\|} \sum_{i, \alpha}\left(S_{i, \alpha}^{+} S_{i+1, \alpha}^{-}+\text {H.c. }\right)-t_{0}^{\perp} \sum_{i}\left(S_{i, 1}^{+} S_{i, 2}^{-}+\text {H.c. }\right)-t_{1}^{\perp} \sum_{i, \alpha}\left(S_{i, \alpha}^{+} S_{i+1, \bar{\alpha}}^{-}+\text {H.c. }\right) \text {. }
$$

Notice that exchanging $t^{\|}$with $t_{1}^{\perp}$ is simply equivalent to renaming the sites $(2 i, \sigma)$ to $(2 i, \bar{\sigma})$ and vice-versa. This is illustrated pictorially in Fig. 5. Thus, a model with $t_{1}^{\perp} / t^{\|}=\tau$ is completely equivalent to one with $t_{1}^{\perp} / t^{\|}=1 / \tau$.

In Fig. 6(a) we plot the finite-size gaps as a function of the system size $L$ for $t_{0}^{\perp}=0$, and different values of $t_{1}^{\perp} / t^{\|}$between 0 and 1 . Given the negligible curvature of the straight-line fits, the data seem to suggest that the gap extrapolates to 0 in all cases. In Fig. 7 we plot the finite-size value of the Luttinger exponent $K_{S}$, determined as explained in sec. VIIB. The data for $K_{S}$ confirm the scenario of a gapless (i.e., rough) system. Notice that $K_{S}$ seems 
to extrapolate to values larger than 1, indicating a rough phase that should survive to the turning on of a suitably small repulsive $V_{1}^{\|}$.

We now address this point in more detail. Consider the $t_{1}^{\perp}=t^{\|}$case (with $t_{0}^{\perp}=0$ ), in which $K_{S}$ seems to extrapolate to the largest value. Denoting by $\left(\begin{array}{c}s \\ s^{\prime}\end{array}\right)$, with $s, s^{\prime}= \pm 1$, the four possible configurations at each site, we can define the following four states:

$$
|\uparrow\rangle=\left|\left(\begin{array}{c}
+1 \\
+1
\end{array}\right)\right\rangle, \quad|\downarrow\rangle=\left|\left(\begin{array}{l}
-1 \\
-1
\end{array}\right)\right\rangle, \quad\left|0_{ \pm}\right\rangle=\frac{1}{\sqrt{2}}\left(\left|\left(\begin{array}{c}
+1 \\
-1
\end{array}\right)\right\rangle \pm\left|\left(\begin{array}{l}
-1 \\
+1
\end{array}\right)\right\rangle\right) .
$$

It is now straightforward to verify that, at each site, the state $\left|0_{-}\right\rangle$is decoupled from the three remaining ones. The Hamiltonian $H$ can then be considered a spin-1 Hamiltonian acting on the subspace spanned by the three states $|\uparrow\rangle,|\downarrow\rangle$, and $\left|0_{+}\right\rangle$. As it can be checked by explicitly calculating all matrix elements, $H$, when restricted within this subspace, coincides with the Heisenberg spin-1 Hamiltonian at $J_{z}=0$ and $J_{x y}=2 t^{\|}$. As argued by den Nijs and Rommelse, 33 the location of the KT transition in the Heisenberg spin-1 phase diagram is, very likely, exactly at $J_{z}=0$. Thus, two coupled XY chains with $t_{1}^{\perp}=t^{\|}$and $t_{0}^{\perp}=0$ have a $K_{S}$ actually equal to 1 , and what we see in Fig. 7 is only due, very likely, to finite-size effects.

The effect of turning on $t_{0}^{\perp}$, while keeping $t_{1}^{\perp}=0$, leads to a completely different picture. In this case, for $t_{0}^{\perp}=t^{\|}$ the system is gapped, as suggested by the finite-size data of Fig. 6(b). The physical reason for the different behavior of the $t_{1}^{\perp}$ and $t_{0}^{\perp}$ terms can be understood by considering the limiting cases of large values for these parameters. For $t_{0}^{\perp} \rightarrow \infty$, the ground state tends to have $\left|0_{+}\right\rangle$at each site, with a large gap (of order $t_{0}^{\perp}$ ) to other exited states. For $t_{1}^{\perp} \rightarrow \infty\left(\right.$ at $\left.t_{0}^{\perp}=0\right)$, on the other hand, the system reduces, by the previously described duality property, to two uncoupled XY chains, and must, therefore, be gapless.

The previous considerations lead us to conjecture that for any choice of $t_{1}^{\perp}$ (as long as $t_{0}^{\perp}=0$ ), two XY chains are gapless and have $K_{S}=1$. On the contrary, turning on $t_{0}^{\perp}$, at $t_{1}^{\perp}=0$, immediately opens up a gap. These conclusions have important consequences on the stability of the rough phase. Since $K_{S}$ attains, at best, the marginal value of 1 , turning on any positive $V_{1}^{\|}$immediately opens up a gap, and the rough phase is confined to infinite temperature.

We will now demonstrate that, if we allow for the possibility of step-crossing events, $t_{e x}>0$, the gapless phase survives the turning-on of a positive $V_{1}^{\|}$, and every "surface" has a rough phase for high enough $T$.

In order to show this, we add to the Heisenberg spin-1 Hamiltonian a $t_{e x}$ term, see Eqs. (7,12),

$$
-t_{e x} \sum_{i}\left[\left(S_{i}^{+}\right)^{2}\left(S_{i+1}^{-}\right)^{2}+\text { H.c. }\right]
$$

with $t_{e x}=t^{\|}=J_{x y}$. Fig. $\mathrm{O}$ shows the phase diagram for this case. Qualitatively, it is very similar to the Heisenberg spin-1 case (see Fig. 3), except for small values of the potentials, where the $t_{e x}$ term changes the structure of the phase diagram. In fact, for $\mu=0$ we observe a gapless phase extending for positive values of $V_{1}^{\|}$, up to $V_{1}^{\|} \approx 0.4$ : this is demonstrated in Fig. 9 were we plot the Luttinger exponent $K_{S}$ along the line $\mu=0$. This finding is in accord with bosonization: the $t_{e x}$ term, unlike the $t^{\perp}$ terms, increases $K_{S}$ and leads to a stabilization of the rough phase. Indeed, for two XY-chains it is easy to show that, up to lowest order in $t_{e x}$,

$$
K_{S}=1+\frac{t_{e x}}{8 \pi^{2} t \|} .
$$

Another remarkable feature of the phase diagram in Fig. 8 is that, at variance with the ordinary Heisenberg case $\left(t_{e x}=0\right)$, the temperature line for a given "surface" crosses the DOF region only if the cost of a step, $\delta_{S}$, is sufficiently small as compared to the interaction between steps, $\widetilde{V}_{1}^{\|}$. We have illustrated this by sketching in Fig. 8 temperaturelines for three different situations. For the case labeled A, the energy cost of a step is high with respect to the interaction energy between steps, and there is no preroughening. In the case labeled $\mathrm{B}, \delta_{S} / \widetilde{V}_{1}^{\|}$is smaller, and a DOF phase is present at intermediate temperatures. Finally, for case C, the interaction between steps is the most relevant energy, and the low temperature phase is $2 \times 1$ reconstructed.

\section{Presence or absence of preroughening: Role of interactions versus step line tension.}

We now want to discuss in some detail what happens if we leave the condition $V_{1}^{\perp}=-V_{1}^{\|}$, without going to the extreme case $V_{1}^{\perp}=0$, discussed in Sec. VIIA. We illustrate this by choosing $V_{1}^{\perp}=-V_{1}^{\|} / 10$, while keeping 
$t_{e x}=t^{\|}=1$ and $V_{0}^{\perp}=\infty$ (infinite on-site repulsion of opposite steps). This choice of parameters describes a class of surfaces in which the attraction between steps of opposite kind is much smaller than the repulsion between steps of the same kind.

In Fig. 10 we plot the phase diagram for this choice of parameters. The system is Néel ordered for very large values of $V_{1}^{\|}$; the value of the ratio $V_{1}^{\|} /\left|V_{1}^{\perp}\right|$ determines the location of the DOF-Néel phase boundary (see Fig. 8 and Fig. 4; recall that, for $V_{1}^{\perp}=0$, the Néel phase is absent for physical values of $\mu$ ). The most relevant comment to the phase diagram in Fig. 10 regards the conditions upon which the temperature trajectories of an actual surface model cross the preroughening line. It is clear, in fact, that depending on the ratio between the cost of a step (per unit length) $\delta_{S}$ and, say, the interaction energy between steps of the same kind $\widetilde{V}_{1}^{\|}$, a surface can have: i) only roughening (case A), or ii) first preroughening and then roughening (case B). Now, with $V_{1}^{\perp}=-V_{1}^{\|} / 10$, the "critical ratio" $\left(\delta_{S} / \widetilde{V}_{1}^{\|}\right)_{\text {crit }}$, below which preroughening is possible is of the order of $1 / 10$, much smaller then in the $V_{1}^{\perp}=-V_{1}^{\|}$case (where $\left(\delta_{S} / \widetilde{V}_{1}^{\|}\right)_{\text {crit }} \approx 1$ ). Given the fact that $\delta_{S}$ is typically the largest "diagonal" energy, this implies that a physical temperature trajectory will be, most likely, in the region where only roughening occurs. If, and how, long-range interactions might change this picture is an interesting and open problem.

\section{Role of opposite-step on-site repulsion: Finite $V_{0}^{\perp}$}

At last, we want to discuss briefly what happens to the DOF phase if we allow double occupation of a site, i.e., if we do not take the limit $V_{0}^{\perp} \rightarrow \infty$. To demonstrate that the restriction to $V_{0}^{\perp}=\infty$ is not crucial, we consider the case $V_{1}^{\|}=-V_{1}^{\perp}=1, t^{\|}=t_{1}^{\perp}=1$, with $V_{0}^{\perp}$ finite. If $V_{0}^{\perp} \rightarrow \infty$ the system is an Heisenberg spin-1 chain at the isotropic point, corresponding to a DOF phase. [3] In Fig. 11 we plot the finite-size values of the flatness order parameter $\mathcal{F}$ (open symbols) and of the DOF correlation function $G_{s}(L / 2)$ (full symbols) for decreasing values of $V_{0}^{\perp}$. The data suggest that the system remains DOF all the way down to $V_{0}^{\perp} \approx 0$. We have verified that a similar scenario is found if we turn on the $t_{e x}$ term or a small $|\mu|$. Thus, our finite-size data suggest that the spin- 1 condition $\left(V_{0}^{\perp}=\infty\right)$ is not essential in order to stabilize a DOF phase.

\section{STEP-STEP CORRELATIONS}

Correlation functions involving steps can be calculated numerically, for a given finite size, at any point in the phase diagram of our model. We will discuss here two correlation functions, i.e., step-step correlations and terrace width distributions. Let $n_{S}$ be the average density of steps of a single species (up or down). In general $n_{S}$ is always different from zero, even in the flat phase, since we do not discriminate between steps that traverse the entire sample and steps that form loops (i.e., finite terraces). Step-step correlations are defined as follows:

$$
N^{\uparrow \sigma}(r)=\frac{1}{n_{S}^{2}}\left\langle\operatorname{Step}^{\uparrow}(0) \operatorname{Step}^{\sigma}(r)\right\rangle=\frac{1}{n_{S}^{2}}\left\langle n_{0, \uparrow} n_{r, \sigma}\right\rangle,
$$

with $\sigma=\uparrow, \downarrow$. If translational symmetry is not broken, we must have, at large distances, $N^{\uparrow \sigma}(r \rightarrow \infty) \rightarrow 1$. The distribution of terrace sizes (along the $x$-direction only!) is the probability of having two steps a distance $r$ apart without any other step in between. There are two different kind of terraces we can look at: those delimited by two steps of the same type, and those between two different steps. Thus, we define:

$$
P^{\uparrow \sigma}(r)=\frac{1}{n_{S}^{2}}\left\langle n_{0, \uparrow}\left[\prod_{j=1}^{r-1}\left(1-n_{j, \uparrow}\right)\left(1-n_{j, \downarrow}\right)\right] n_{r, \sigma}\right\rangle,
$$

where, again, $\sigma=\uparrow, \downarrow$. The string operator in square brackets enforces the absence of additional steps between 0 and $r$. Fig. 12 illustrates the behavior of $N^{\uparrow \uparrow}$ and $N^{\uparrow \downarrow}$, at three different points in the phase diagram of the Heisenberg spin-1 chain: a rough case $\left(J_{z}=-0.5, \mu=0\right.$, triangles), a DOF case $\left(J_{z}=1\right.$ and $\mu=0$, squares), and a flat one ( $J_{z}=1$ and $\mu=-2$, pentagons). The flat case results are very simple: both $N^{\uparrow \uparrow}$ and $N^{\uparrow \downarrow}$ converge exponentially fast (with a very short correlation length) to the large distance limit of 1 . In the rough phase, instead, we have verified that the approach to 1 shows a power law tail. This is easy to prove. Rewrite first $N^{\uparrow \sigma}$ in terms of density and "spin" correlations:

$$
N^{\uparrow \sigma}(r)=\frac{1}{4 n_{S}^{2}}\left[\left\langle n_{0} n_{r}\right\rangle \pm\left\langle S_{0}^{z} S_{r}^{z}\right\rangle\right]
$$


where the + and - signs apply, respectively, to $\sigma=\uparrow$ and $\sigma=\downarrow, n_{i}=n_{i, \uparrow}+n_{i, \downarrow}$, and $S_{i}^{z}=n_{i, \uparrow}-n_{i, \downarrow}$. Within a bosonization approach, [23] the operators $n_{i}$ and $S_{i}^{z}$ involve (after particle-hole transformation for the $\downarrow$-bosons) only the antisymmetric and symmetric sectors, respectively. The antisymmetric sector is always gapped (see discussion in Sec. $\mathrm{V}$ and Ref. [23]), so that density-density correlations are exponential. In the rough phase, however, the symmetric sector is gapless, and $S^{z}-S^{z}$ correlations have a uniform power-law tail of the form

$$
\left\langle S_{0}^{z} S_{r}^{z}\right\rangle=-\frac{K_{S}}{(\pi r)^{2}}+\cdots,
$$

which is precisely the term responsible for the logarithm in the heigth-heigth correlation function $G(r)=\left\langle\left(h_{r}-h_{0}\right)^{2}\right\rangle$. 25] The DOF case results, finally, show a different behavior, with a sizeable oscillating component of the correlations. This behavior, however, reflects only a short-range effect, caused by the neighboring reconstructed (Néel) phase: the oscillating part has to decrease to zero at large $r$, since no breaking of translational symmetry occurs in the DOF phase. 2]

We finally discuss briefly the behavior of the distributions of terrace sizes (for simplicity, once again, in the Heisenberg spin-1 case). While in principle it is important to know what is the probability for the surface to be flat over a distance $r$, this quantity has never been calculated so far.

Let us consider, first, the behavior of $P^{\uparrow \downarrow}$ in the rough phase. Fig. 13(a) is a plot of the logarithm of $P^{\uparrow \downarrow}$ versus a scaled distance $2 n_{S} r$, for several points taken inside the rough phase of the Heisenberg spin-1 phase diagram. We observe that the general behavior of $P(r)$ is exponential in the size of the terrace $r$,

$$
P(r) \approx e^{-r / \lambda}
$$

and that a good collapse is obtained for all data if the distance $r$ is scaled to the average separation between two steps, $1 /\left(2 n_{S}\right)$, i.e., $\lambda \propto 1 /\left(2 n_{S}\right)$. The scattering of the data for the largest $r$ 's is due to finite-size effects. The behavior of $P^{\uparrow \uparrow}(r)$ is found to be qualitatively similar.

In the DOF phase we find that the terrace size distribution probability is again exponential with size, but now $\lambda$ does not scale with the density of steps, as it did instead in the rough phase. Fig. 13(b) illustrates the behavior of $P(r)$ at a DOF point, corresponding to the isotropic Heisenberg point of the spin-1 chain. $P(r)$ at a rough point is also reported for comparison. We observe that, as anticipated, the behavior of $P^{\uparrow \sigma}$ is, once again, exponential in $r$ (i.e., the "DOF checkerboard" has no typical length!). Superimposed on the leading exponential, the DOF case results show a strong oscillating short range component which is again due to the neighboring reconstructed (Néel) phase. Two more features are worth noticing. First, compared to the rough case, $P^{\uparrow \downarrow}(r)$ is larger in the DOF case for $r=1$, and then substantially smaller for larger values of $r$ (and decreasing with a larger exponent). Second, in the DOF case $P^{\uparrow \uparrow}(r)$ is one order of magnitude smaller than $P^{\uparrow \downarrow}(r)$, while the difference is much smaller in the rough case. These features are reasonable in view of the diluted antiferromagnetic ordering of steps, typical of the DOF phase.

Experimentally, terrace sizze distributions could in the future be extracted, e.g., from STM data. [26]

\section{SUMMARY AND CONCLUSIONS}

In this paper we have presented and discussed a statistical mechanics model for studying the possible phase transitions of an ideal, unreconstructed surface. The elementary objects upon which the model is based are the natural extended defects of an unreconstructed surface, i.e., steps and terraces. This starting point is, in our opinion, physically more transparent then the usual microscopic RSOS-model description. Our model allows, in principle, the description of a real surface and, in perspective, one could test it with realistic step-step interactions.

We have tackled our problem of interacting steps by mapping it, in a well known way, onto a one dimensional quantum problem of interacting hard-core bosons. Although this mapping is exact only in the strong anisotropy limit, it can provide very useful information about the phases and the nature of the transitions also in more general instances. Moreover, some realistic cases, like (110) surfaces of fcc metals, are actually quite anisotropic.

The quantum Hamiltonian, see Eq. (7), contains standard terms, like nearest-neighbor hopping (describing kinks on the steps), potential terms (describing interactions between steps), and chemical potential (cost per unit length of a step), as well as terms describing i) terrace creation/annihilation (through BCS-like number non conserving terms), ii) opposite step crossing events. The latter two terms are crucial, in many ways.

Terrace terms are important to describe correctly the universality classes of the relevant transitions. This is known in the literature, [27, 16, 15] but never explored in details in the present context. Moreover, in our case, the terrace terms also force us to work with hard-core bosons, as the standard Wigner-Jordan transformation to fermions does not lead to a simple local fermionic Hamiltonian. This point is sometimes overlooked in the literature. [28] 
The term describing the crossing of opposite steps is important in order to stabilize a gapless (i.e., rough) phase for finite repulsive interactions between steps of the same kind. This, in turn, leads to a finite roughening temperature for the classical model.

Finite-size exact diagonalizations and bosonization techniques have been used to unveil the richness of the phase diagram. In the limit of $V_{0}^{\perp} \rightarrow \infty$ and for a particular choice of parameters (the potentials, for instance, are truncated to first neighbors and set to $\left.V_{1}^{\|}=-V_{1}^{\perp}\right)$, the model maps exactly onto the Heisenberg spin-1 chain Hamiltonian. The latter was also obtained, by den Nijs and Rommelse as the quantum mapping of RSOS models; [3] it presents a DOF phase for $V_{1}^{\|}>0$, but does not describe, in that case, a surface with a finite temperature roughening. (On the other hand, if $V_{1}^{\|}$is attractive there is only roughening).

Taking the Heisenberg chain as a starting point, we have then explored the phase diagram for other choices of parameters, obtaining results that we believe to be relevant with respect to the surface physics interpretation of our model. Summarizing, we have seen that:

1. The Heisenberg spin-1 restriction $V_{1}^{\|}=-V_{1}^{\perp}$ is not crucial in stabilizing the DOF phase. In particular, we observe a DOF phase even for $V_{1}^{\perp}=0$ (see Sec. VII A). Moreover, a DOF phase is present not only for $V_{0}^{\perp}=\infty$ (spin-1 case) but also when $V_{0}^{\perp}$ is finite, as long as positive.

2. If we add to the Heisenberg spin-1 Hamiltonian a $t_{e x}$ term, we observe a gapless phase extending for positive values of $V_{1}^{\|}$. Every surface has a rough phase for high enough $T$. This is true also for other choices of the potentials (see Sec. VIIC). Moreover, if we do not include in the Hamiltonian the $t_{e x}$ term, the rough phase does not survive when one turns on a $V_{1}^{\|}>0$ (see sec. VIIB). Thus, the opposite step crossing term is crucial in order to obtain a model describing, at least at a coarse grained level, a physical surface.

3. The relative values of the interactions and of the cost per unit length of a step decide whether a surface has a stable DOF phase for a certain range of $T$. The temperature trajectory crosses the DOF region only if the cost of a step, $\delta_{S}$, is sufficiently small as compared to $\widetilde{V}_{1}^{\|}$(see Fig. 8). Given the fact that $\delta_{S}$ is typically the largest "diagonal" energy, this implies that a physical temperature trajectory will often be in a region where only roughening occurs.

In conclusion, we have found that: (i) a model based on steps can describe preroughening (PR), as well as roughening; (ii) the steps must be treated as hard-core bosons rather than fermions; (iii) the qualitative role of step-step interactions in driving PR, known already from RSOS models, is recovered in this picture; (iv) correlation functions involving steps can be calculated in a quite straightforward way. The main one, never studied so far, which we have considered, is the terrace size distribution. Here we find simply an exponentially decreasing probability for increasing size. This result should be amenable to experimental testing, for example by STM; (v) In view of the additional simplicity of step models, it should be feasible, in the future, to study the role of long-ranged interactions, a problem without hope of solution within RSOS models.

ACKNOWLEDGMENTS - We thank M. Fabrizio, A. Parola, S. Sorella, and F.D.M. Haldane for many useful discussions. We acknowledge financial support from INFM, through Projects LOTUS and HTSC, from EU, through ERBCHRXCT940438, and from MURST, through COFIN97.

[1] For a review see, for instance, J. D. Weeks, in Ordering in Strongly Fluctuating Condensed Matter System, NATO ASI B50, ed. T. Riste (Plenum, New York, 1979); or J. Lapujoulade and B. Salanon, in Phase Transitions in Surface Films 2, Eds. H. Taub et al. (Plenum, New York, 1991).

[2] K. Rommelse and M. den Nijs, Phys. Rev. Lett. 59, 2578 (1987).

[3] M. den Nijs and K. Rommelse, Phys. Rev. B 40, 4709 (1989).

[4] J. A. Jaszczak, W. F. Saam, and B. Yang, Phys. Rev. B 39, 9289 (1989); ibid. 41, 6824 (1990).

[5] G. Mazzeo, G. Jug, A. C. Levi, and E. Tosatti, Surf. Sci. 273, 237 (1992); Europhys. Lett. 22, 39 (1993).

[6] G. Santoro and M. Fabrizio, Phys. Rev. B 49, 13886 (1994). G. Santoro, M. Vendruscolo, S. Prestipino, and E. Tosatti, Phys. Rev. B 53, 13169 (1996).

[7] P. B. Weichman, P. Day, and D. Goodstein, Phys. Rev. Lett. 74, 418 (1995).

[8] S. Prestipino, G. Santoro, and E. Tosatti, Phys. Rev. Lett. 75, 4468 (1995).

[9] P. J. M. Bastiaansen and H. J. F. Knops, Phys. Rev. B, 53, 126 (1996). 
[10] H. S. Youn and G. B. Hess, Phys. Rev. Lett. 64, 918 (1990).

[11] P. Day et al., Phys. Rev. B 47, 7501 (1993); Phys. Rev. B 47, 10716 (1993); H. S. Youn, X. F. Meng, and G. B. Hess, Phys. Rev. B 48, 14556 (1993).

[12] J. Villain and I. Vilfan, Surf. Sci. 199, 165 (1988).

[13] J. Villain and I. Vilfan, Europhys. Lett. 12, 523 (1990); ibid. 13, 285 (1990).

[14] I. Vilfan and J. Villain, Surf. Sci. 257, 368 (1991).

[15] M. den Nijs, Phys. Rev. B 46, 10386 (1992).

[16] L. Balents and M. Kardar, Phys. Rev. B 46, 16031 (1992).

[17] J. Villain, D. R. Grempel, and J. Lapujoulade, J. Phys. F: Met. Phys. 15809 (1985).

[18] J. B. Kogut, Rev. Mod. Phys. 51, 659 (1979).

[19] M. den Nijs, in Phase transitions and critical phenomena Vol. 12, p. 219, ed. C. Domb and J. L. Lebowitz (Academic Press, London, 1988).

[20] H. J. Schulz, B. I. Halperin, and C. L. Henley, Phys. Rev. B 26, 3797 (1982).

[21] Y. Hatsugai and M. Kohmoto, Technical Report of ISSP, Ser. A, No. 2443 (1991).

[22] For a vicinal surface of angle $\phi$, we would have $\sum_{i}\left(S_{i, 1}^{z}+S_{i, 2}^{z}\right)=L \tan \phi$.

[23] S. P. Strong and A. J. Millis, Phys. Rev. B 50, 911 (1994).

[24] F. D. M. Haldane, J. Phys. C:Solid State Phys., 14, 2585-2609 (1981)

[25] With the notation $S_{i}^{z}=n_{i \uparrow}-n_{i \downarrow}$, the height difference between site $r$ and site 0 is given by the sum $\sum_{i=1}^{r-1} S_{i}^{z}$. Thus, using translational invariance for the spin-spin correlation function,

$$
G_{h}(r)=\sum_{i, j=1}^{r-1}\left\langle S_{i}^{z} S_{j}^{z}\right\rangle=r\left\langle\left(S_{0}^{z}\right)^{2}\right\rangle+2 \sum_{j=1}^{r-1}(r-j)\left\langle S_{0}^{z} S_{j}^{z}\right\rangle \approx-2 \sum_{j=1}^{r-1} j\left\langle S_{0}^{z} S_{j}^{z}\right\rangle
$$

If the uniform part of the spin-spin correlation function $\left\langle S_{0}^{z} S_{j}^{z}\right\rangle$ behaves, for large $j$ 's, like $\frac{-K}{4 \pi^{2} j^{2}}, G_{h}(r)$ will diverge logarithmically as $\frac{2 K}{\pi^{2}} \ln (r)$.

[26] M.S. Hoogeman, D.C. Schlösser, J.B. Sanders, L. Kuipers, J.W.M. Frenken, Phys. Rev. B 53, R13299 (1996); M.S. Hoogeman, Thesis, Univ. of Leiden (1998).

[27] T. Bohr, Phys. Rev. B 25, 6981 (1982); 265257 (Erratum).

[28] M. Kardar and R. Shankar, Phys. Rev. B 31, 1525 (1985), for instance, consider a problem of domain walls in the context of commensurate-to-incommensurate transitions of adsorbate layers, and set up a model including dislocation terms (exactly analogous to our $t_{1}^{\perp}$ term), in absence of interactions, but solve the model through a Bogoliubov transformation, treating the particles as fermions, rather than hard-core bosons, as we believe one should, as the anticommuting of steps seems devoid of physical significance.

\section{FIGURE CAPTIONS}

FIG. 1. Scheme of a surface with up $(\uparrow)$ and down $(\downarrow)$ steps. The heights of the terraces are explicitly indicated. The other symbols refer to the Boltzmann weights considered: $\delta_{K}$ (cost of a kink), $\delta_{T}$ (cost of a terrace creation), $\delta_{e x}$ (cost of a step crossing), $V^{\|}$and $V^{\perp}$ (interactions between parallel and opposite steps); the black dots indicate where terraces are created or destroyed.

FIG. 2. Schematic representation of a kink, the beginning of a size-1 terrace, a size- 0 terrace, and a step crossing between strip $j$ and strip $j+1$ and the relative energetic costs.

FIG. 3. Phase diagram for the spin-1 Heisenberg chain. [3] Here and in the following we consider only negative values of the chemical potential $\mu$, that are relevant for the surface physics problem. Lines (a) and (b) are discussed in the text.

FIG. 4. Qualitative phase diagram for a spin-1 chain with $t_{1}^{\perp}=t^{\|}=1, t_{e x}=0, V_{1}^{\perp}=0$.

FIG. 5. The duality mapping for two XY chains, see Eq. (15). Dotted, solid and dashed lines denote, respectively, $t_{1}^{\perp}, t^{\|}$, and $t_{0}^{\perp}$ couplings. The duality survives also in presence of $V_{0}^{\perp}$. 
FIG. 6. (a) Finite-size charge excitation gaps for the two XY chains in Eq. (15) at $t_{0}^{\perp}=0, t^{\|}=1$, and for various values of $t_{1}^{\perp}$. Dashed lines are obtained from straight lines constructed so as to pass through the $L=6$ and $L=8$ points. The extrapolation to zero is remarkably good. (b) Finite-size charge excitation gaps for $t_{0}^{\perp}=0, t_{1}^{\perp}=t^{\|}=1$ (dashed line), and $t_{1}^{\perp}=0, t_{0}^{\perp}=t^{\|}=1$ (solid line). The solid and dashed straight lines are constructed as in (a). Notice the remarkable smallness of size corrections.

FIG. 7. Luttinger exponent $K_{S}$ for the same parameters of Fig. 6(a). As argued in the text (see sec. VIIB), $K_{S}$ should converge to 1 for $L \rightarrow \infty$ and the apparent extrapolation to values larger than 1 is very likely due to finite size effects.

FIG. 8. Qualitative phase diagram for a Heisenberg spin-1 chain with exchange term, i.e., $t_{1}^{\perp}=t^{\|}=t_{e x}=1, V_{1}^{\|}=-V_{1}^{\perp}$. Lines (A), (B), and (C) are discussed in the text.

FIG. 9. Finite-size Luttinger exponent $K_{S}$ along the line $\mu=0$, for the Heisenberg spin-1 chain plus exchange term. $K_{S}$ extrapolates to values larger than 1 for $V_{1}^{\|}<0.4$. Dashed lines are only guides to the eye.

FIG. 10. Qualitative phase diagram for a spin-1 chain with $t_{1}^{\perp}=t^{\|}=t_{e x}=1, V_{1}^{\perp}=-V_{1}^{\|} / 10$. Line (A) describes a situation where the cost of a step $\delta_{S}$ is larger than the interactions, and only roughening is found. Line (B) describes a situation where $\delta_{S}$ is smaller than $\left|\widetilde{V}_{1}^{\perp}\right|$, and roughening is preceded by PR.

FIG. 11. Finite-size values of the flatness order parameter $\mathcal{F}$ (open symbols) and of the DOF correlation function $G_{s}(L / 2)$ (full symbols) at the Heisenberg isotropic point, for decreasing values of $V_{0}^{\perp}$. The system appears to be DOF for all positive values of $V_{0}^{\perp}$. The dashed lines are only guides to the eye.

FIG. 12. $\quad N^{\uparrow \downarrow}$ (above) and $N^{\uparrow \uparrow}$ (below), at three different points in the phase diagram of the Heisenberg spin-1 chain: a rough case $\left(J_{z}=V^{\perp}=-V_{1}^{\|}=-0.5, D=-\mu=0\right.$, triangles $)$, a DOF case $\left(J_{z}=V^{\perp}=-V_{1}^{\|}=1\right.$, and $D=-\mu=0$, squares), and a flat one $\left(J_{z}=V^{\perp}=-V_{1}^{\|}=1\right.$, and $D=-\mu=2$, pentagons $)$. Lines are only guides to the eye.

FIG. 13. (a) $\ln \left(P^{\uparrow \downarrow}\right)$ versus the scaled distance $2 n_{S} r$ at various points in the rough phase of the Heisenberg spin-1 chain; full symbols: $J_{z}=V^{\perp}=-V_{1}^{\|}=-0.25, D=-\mu=0,0.1,0.2,0.3$; empty symbols: $J_{z}=V^{\perp}=-V_{1}^{\|}=-0.5, D=-\mu=0,0.2,0.4$; stars: $J_{z}=V^{\perp}=-V_{1}^{\|}=-0.75, D=-\mu=0,0.1,0.2,0.3$. (b) $P^{\uparrow \downarrow}$ and $P^{\uparrow \uparrow}$, in logarithmic scale, at two different points in the phase diagram of the Heisenberg spin-1 chain: a DOF case $\left(J_{z}=V^{\perp}=-V_{1}^{\|}=1\right.$, and $D=-\mu=0$, squares $)$, and a rough case $\left(J_{z}=V^{\perp}=-V_{1}^{\|}=-0.5, D=-\mu=0\right.$, triangles). Full and empty symbols correspond to $P^{\uparrow \downarrow}$ and $P^{\uparrow \uparrow}$, respectively. 


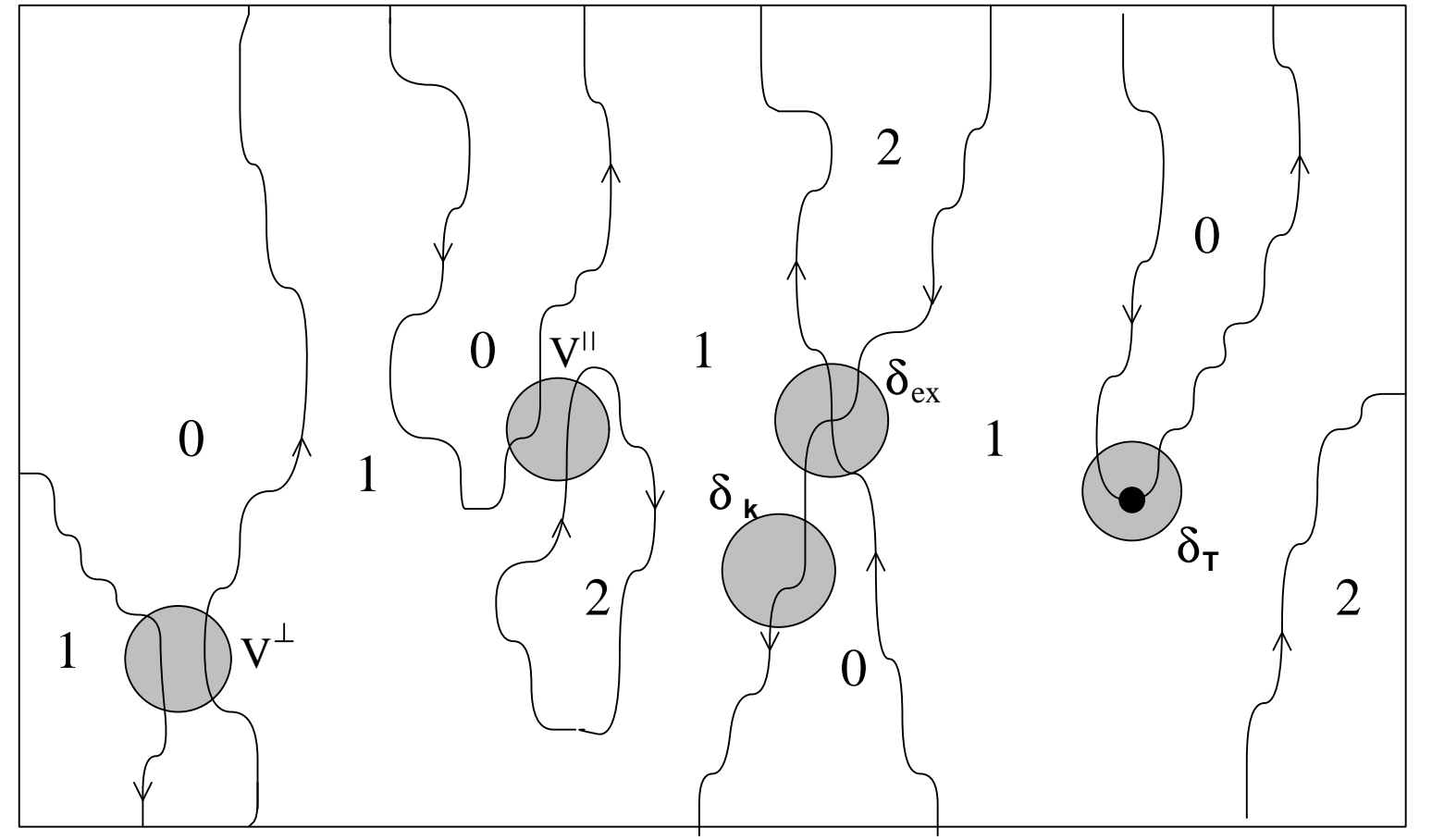




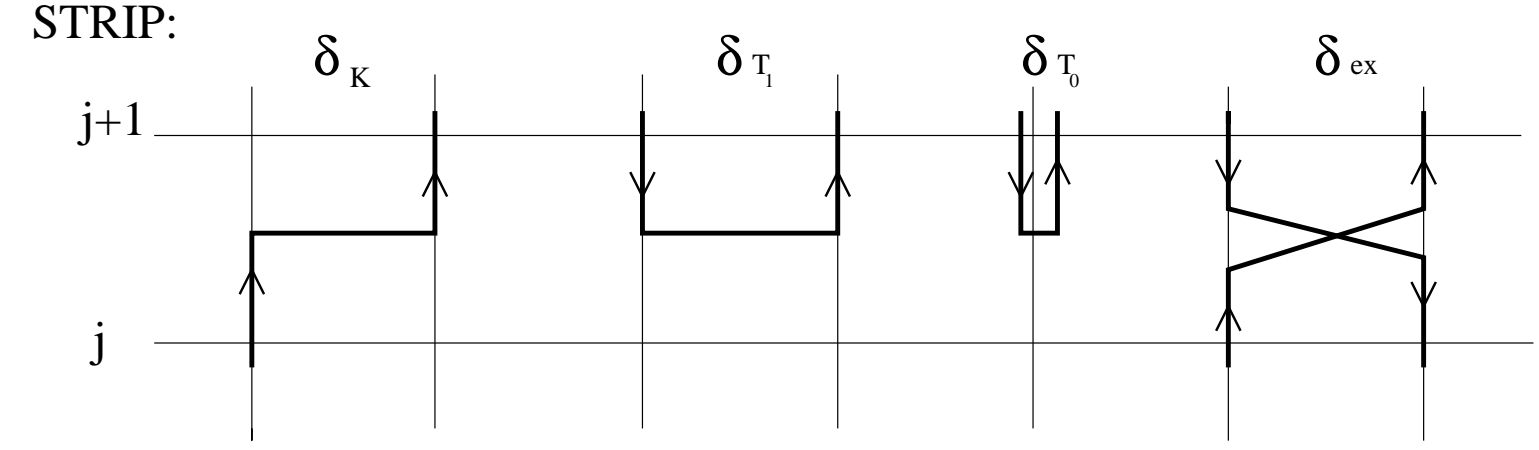


(b)

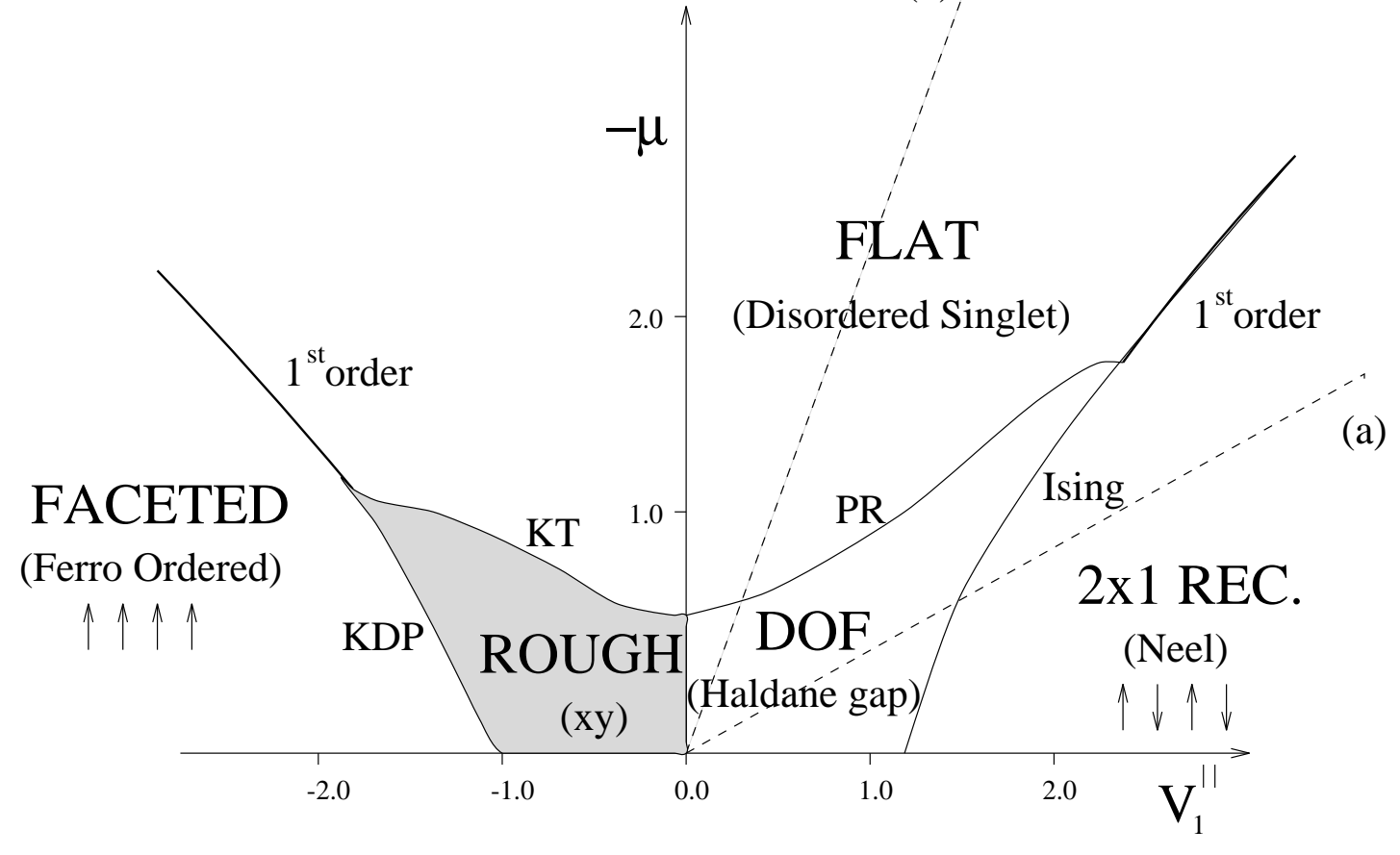




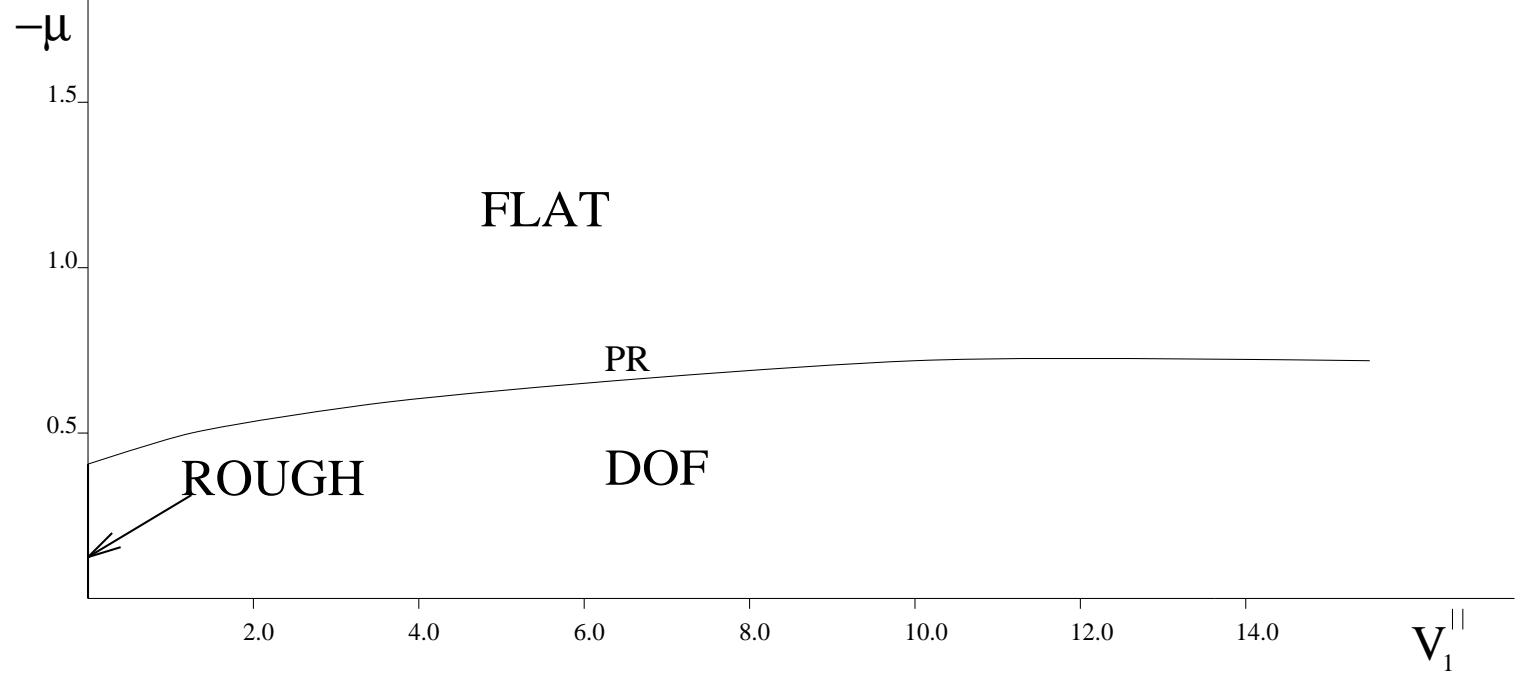


i-1,2 i , 2 i+1,2 $\quad$ i $+2,2$

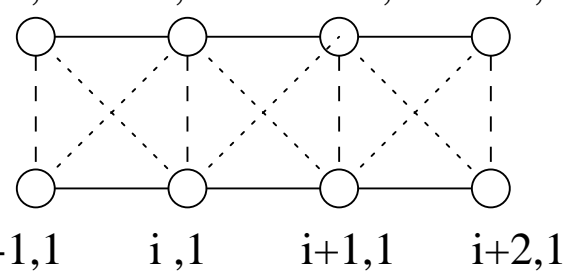

$\mathrm{i}-1,2 \quad \mathrm{i}, 1 \quad \mathrm{i}+1,2 \quad \mathrm{i}+2,1$
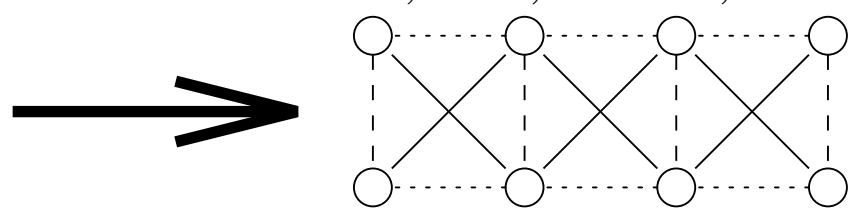

i- $1,1 \quad$ i, $2 \quad i+1,1 \quad i+2,2$ 


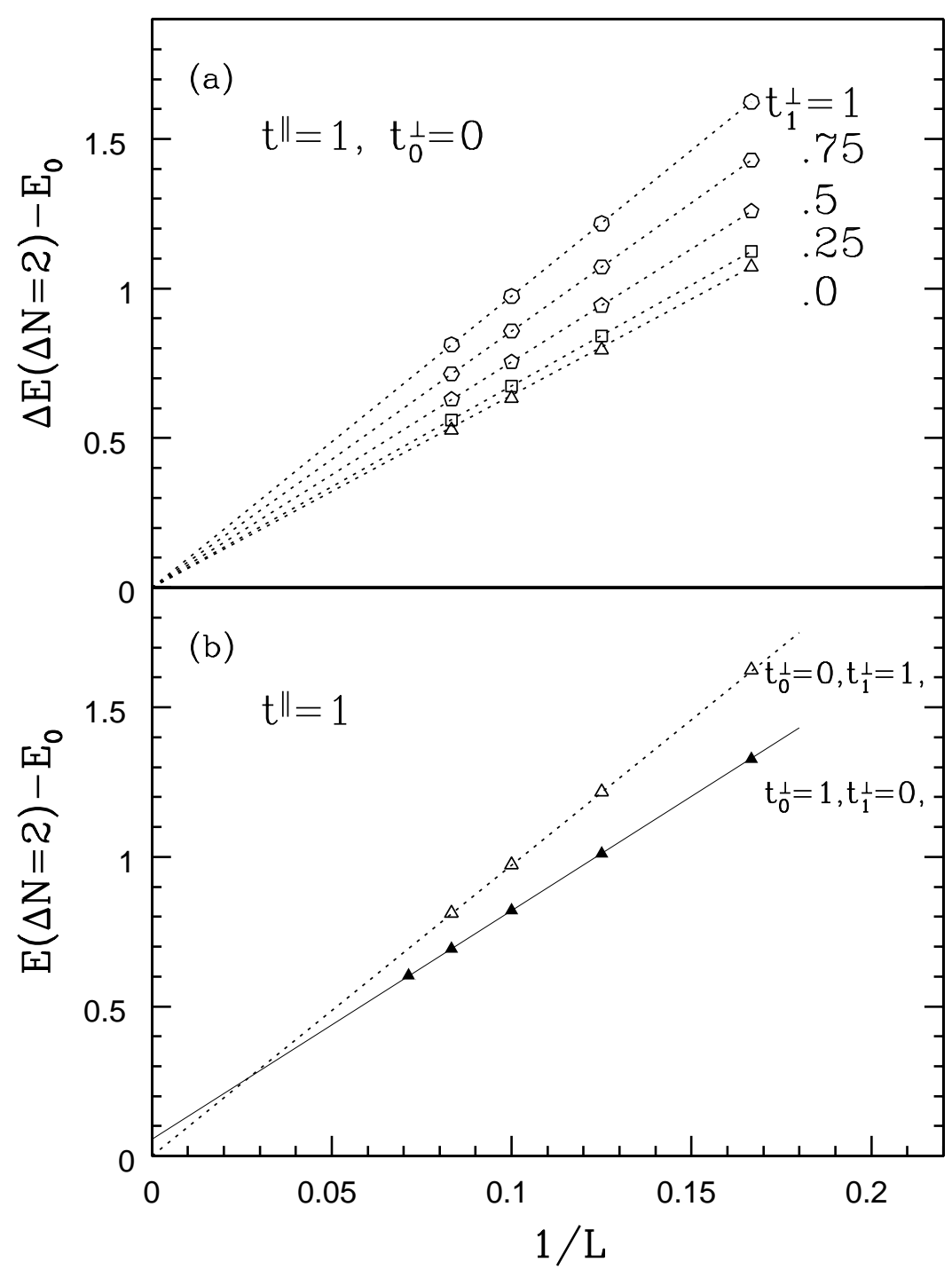




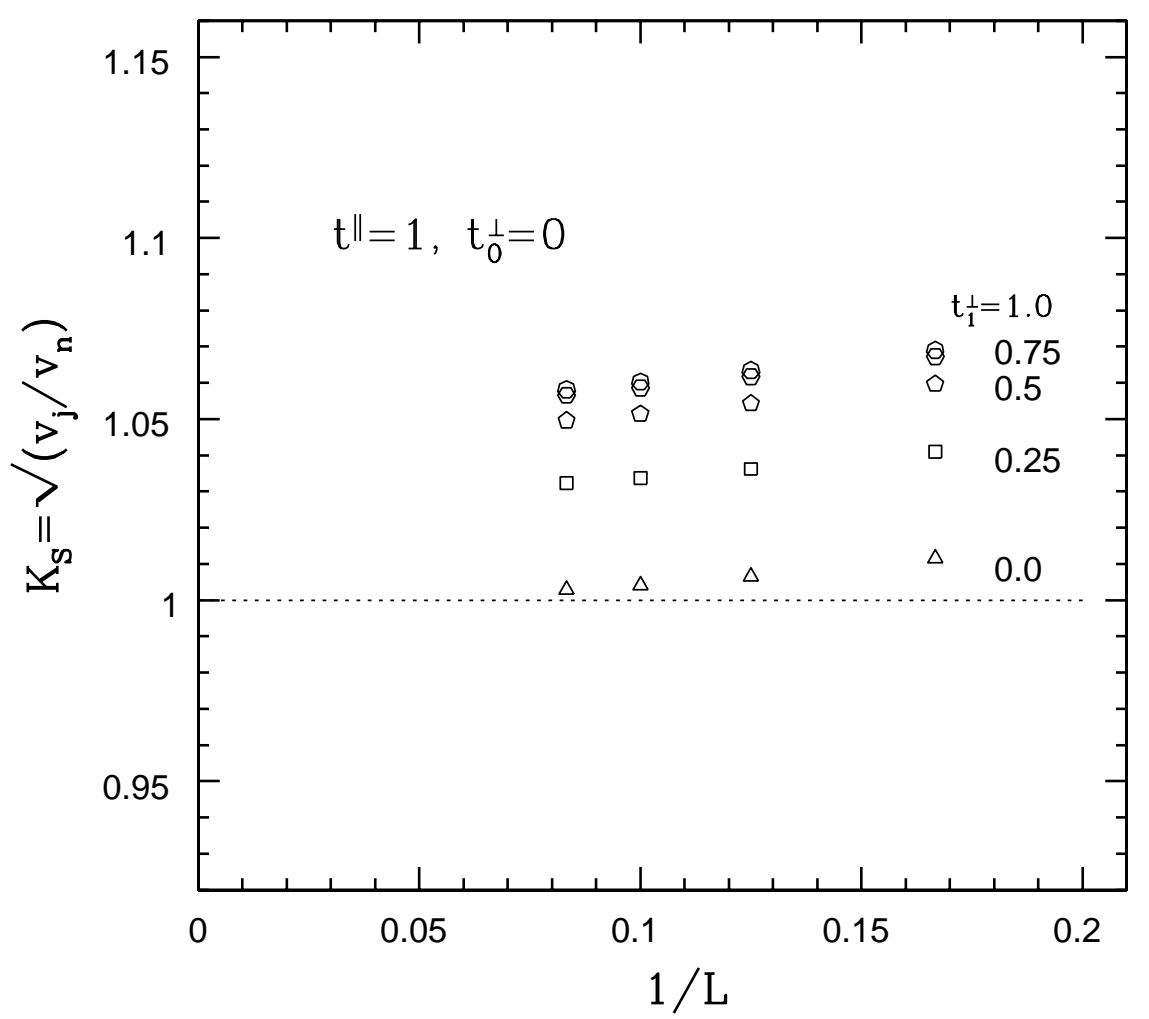




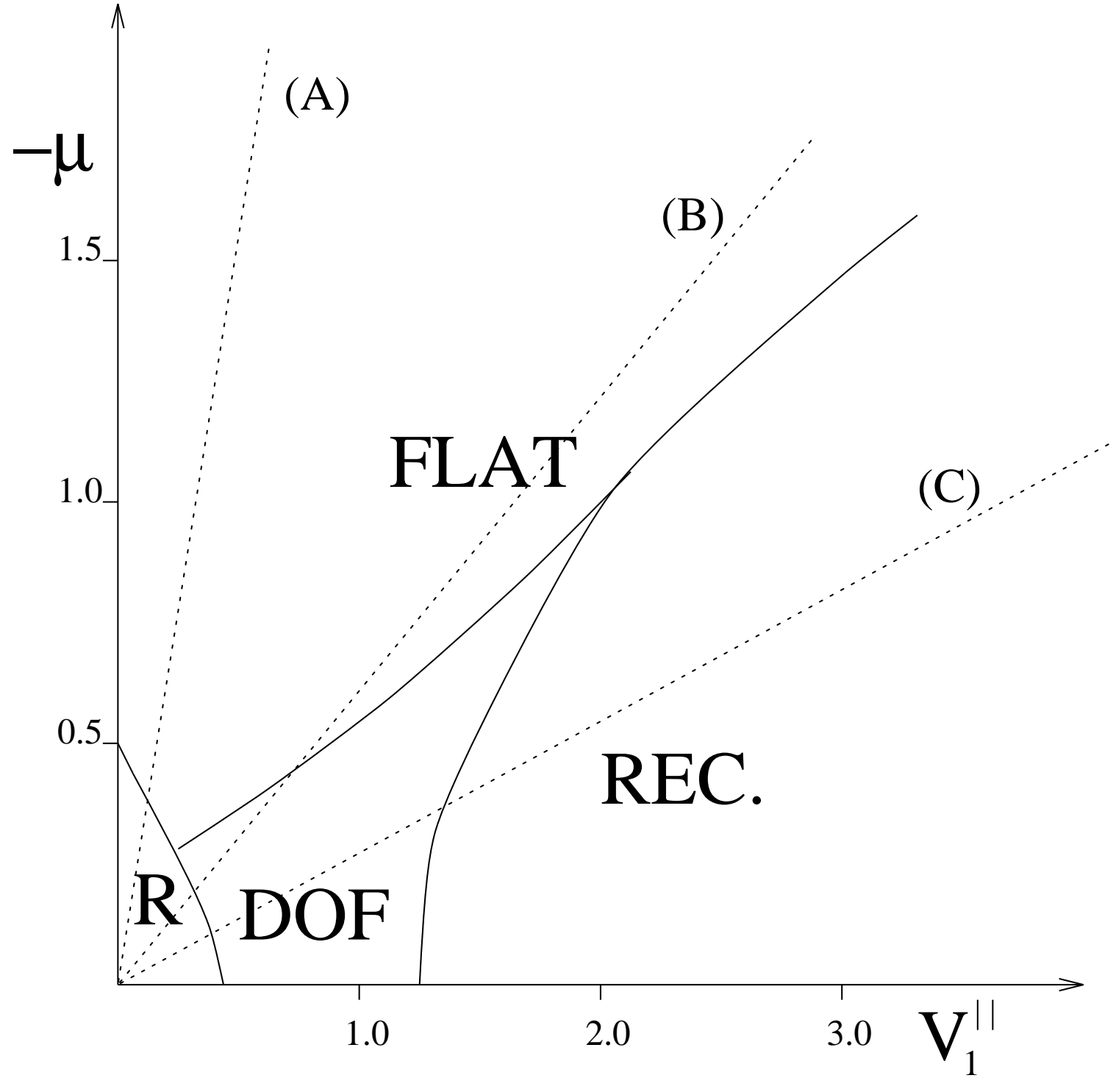




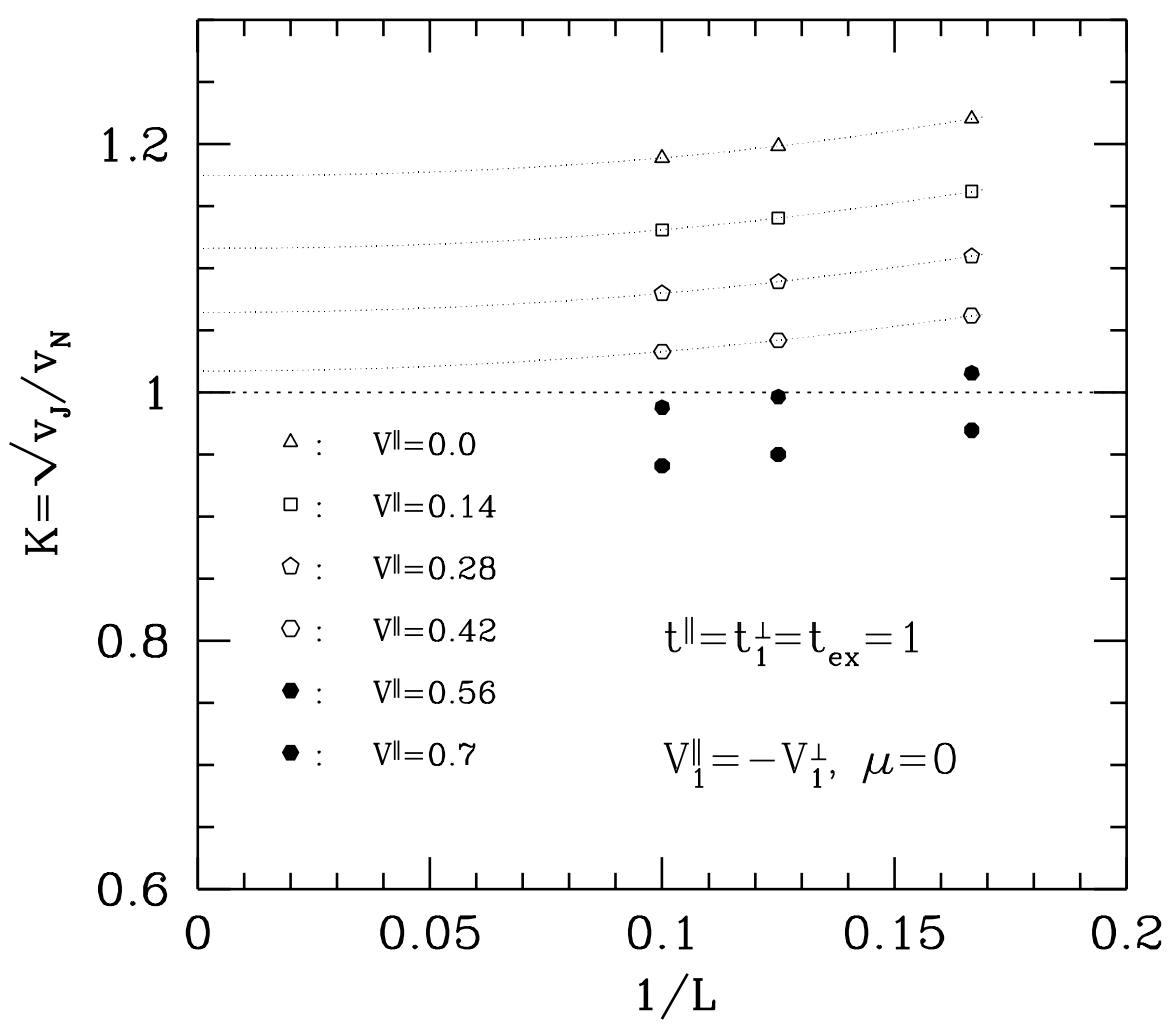




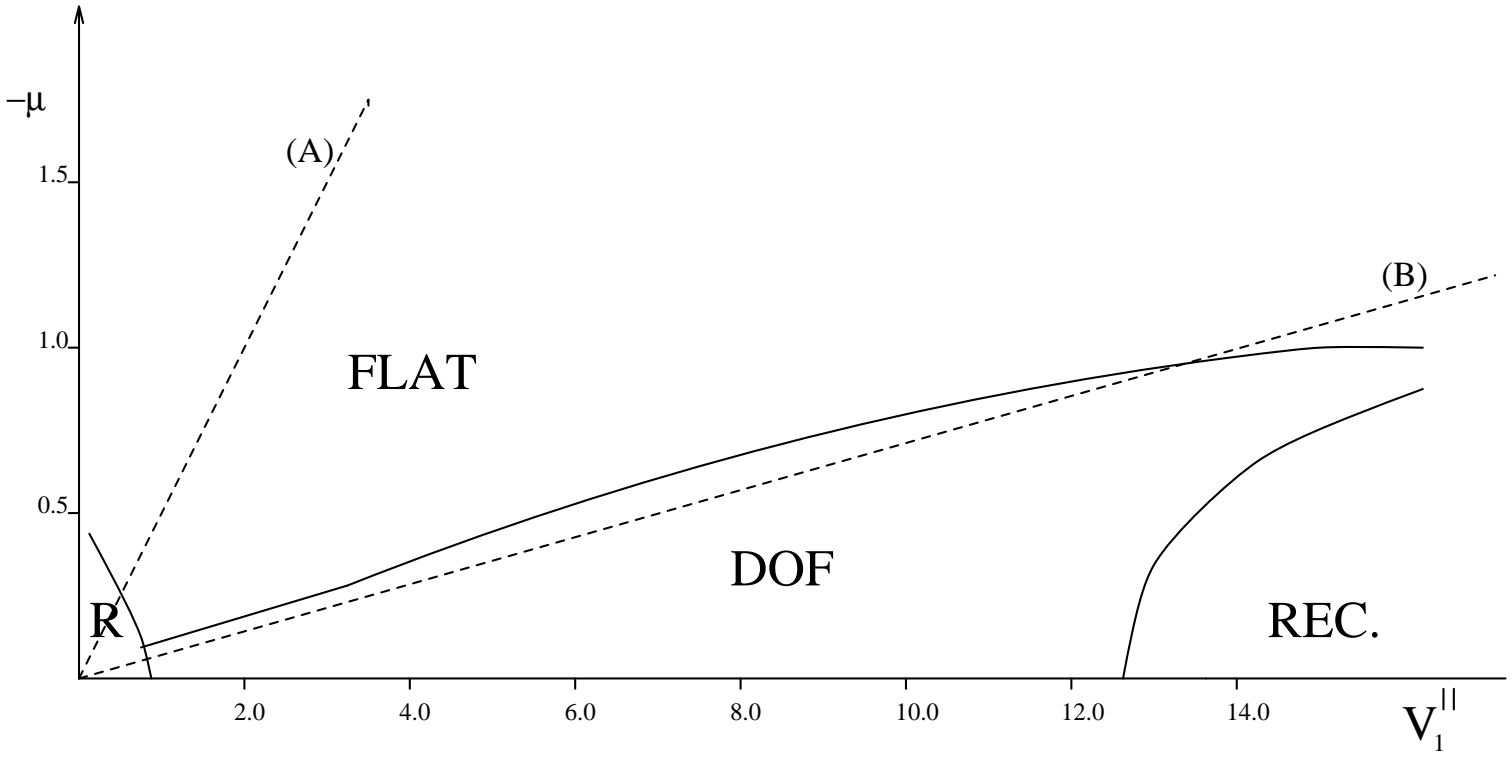




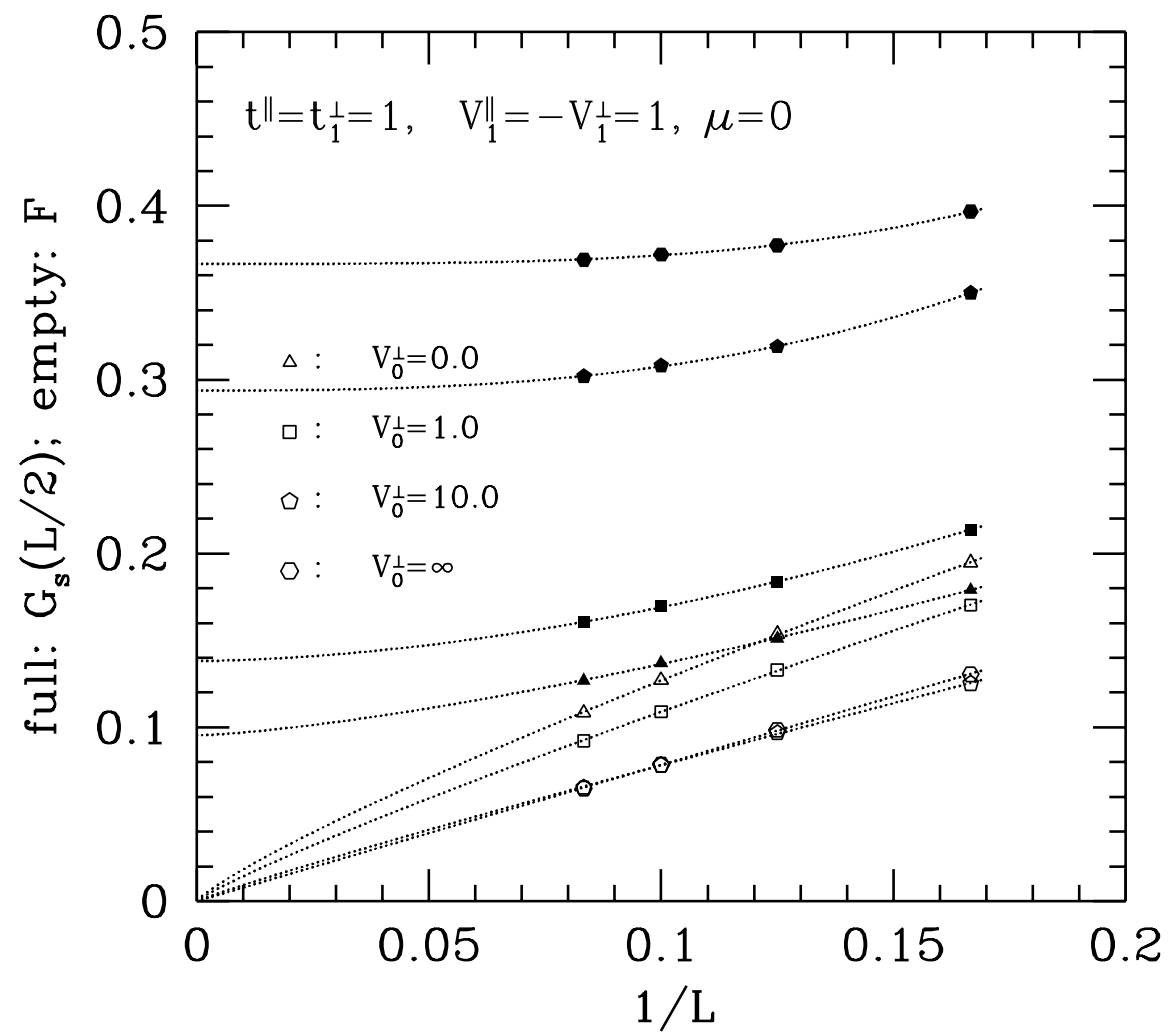




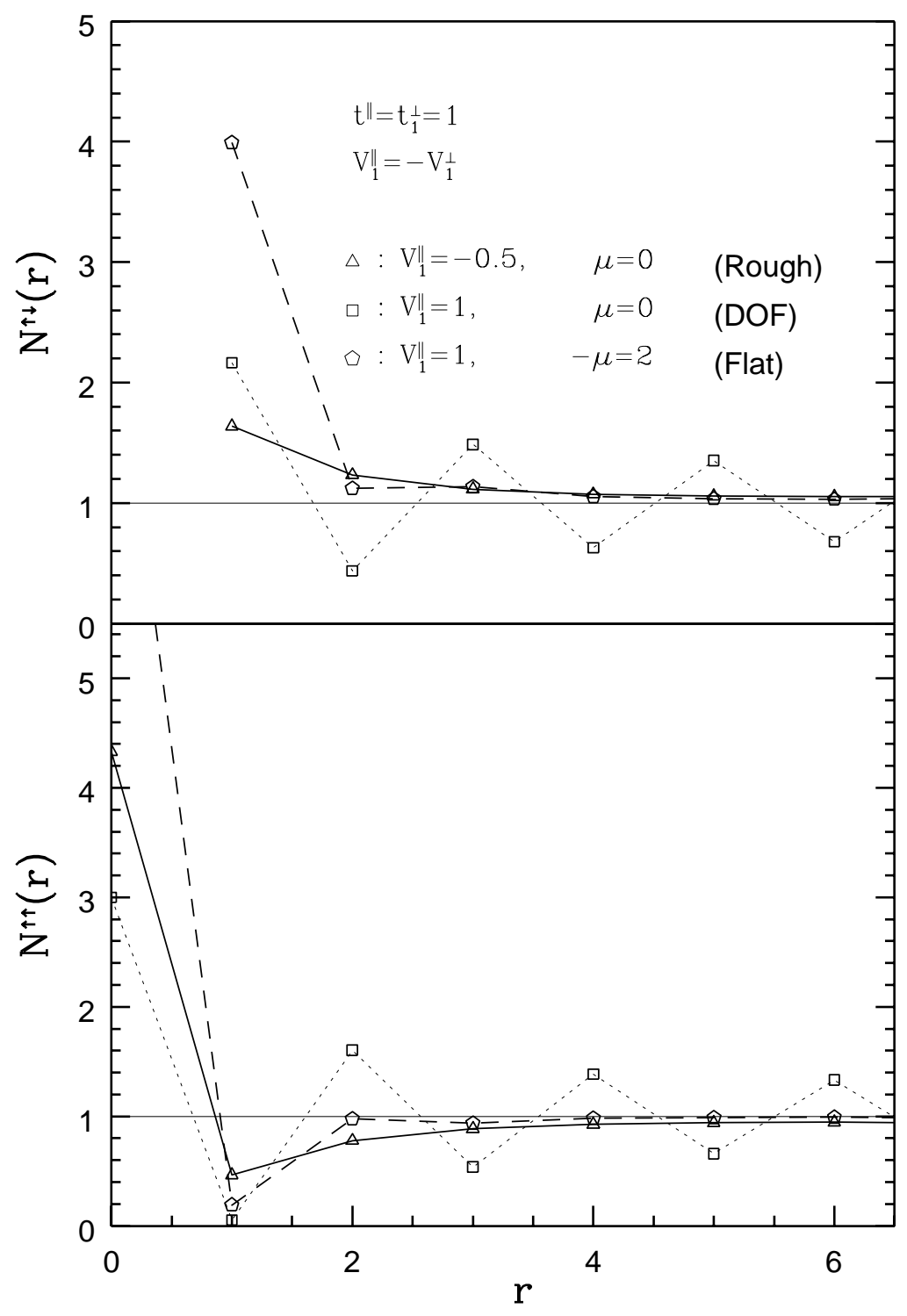



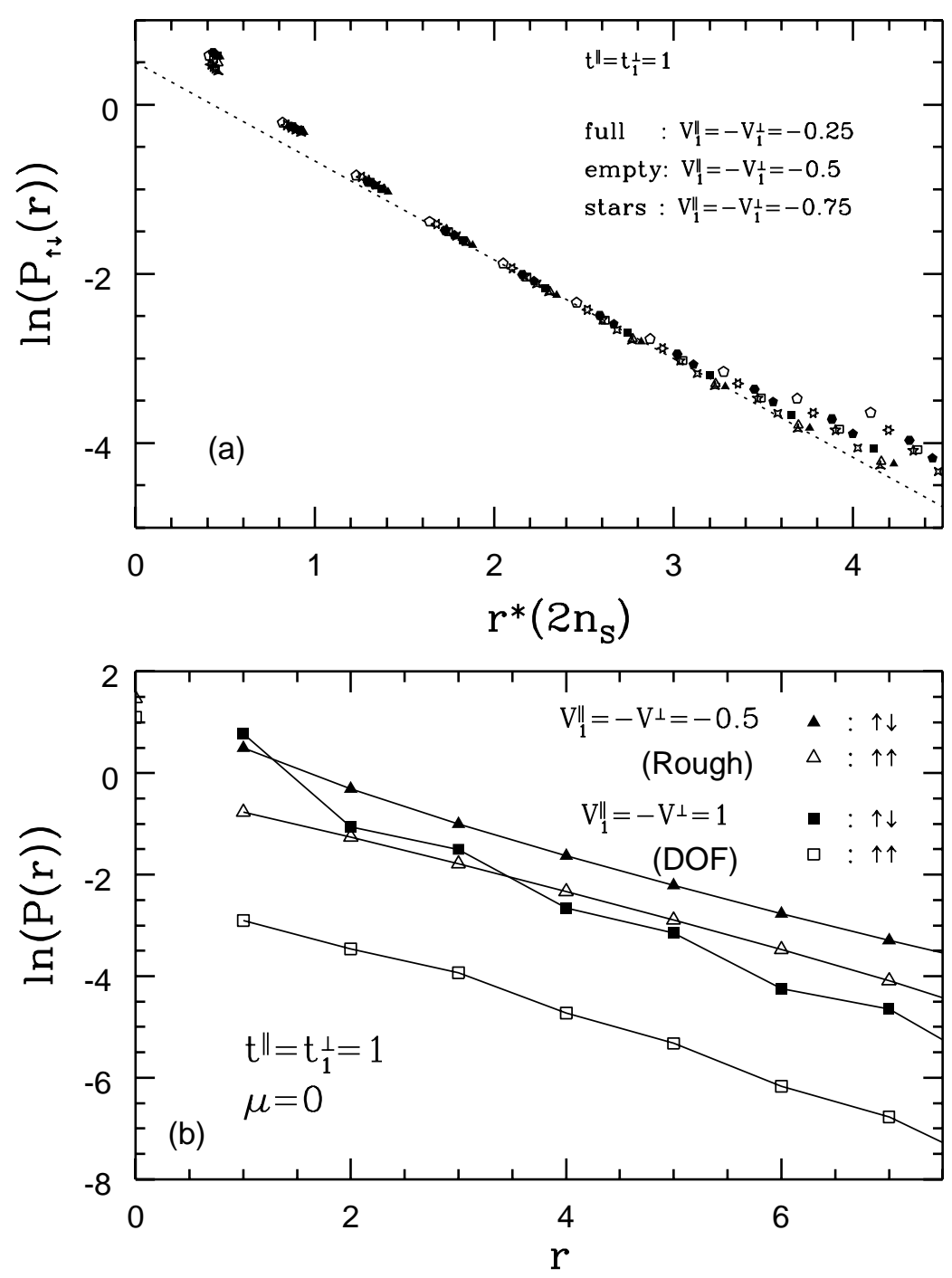